\title{
Adaptive Parameter Modulation of Deep Brain Stimulation in a Computational Model of Basal Ganglia-thalamic Network
}

\section{Yulin Zhu}

Tianjin University

Jiang Wang

Tianjin University

Siyuan Chang

Tianjin University

Huiyan Li

Tianjin University of Technology and Education

\section{Bin Deng}

Tianjin University

Chen Liu ( $\boldsymbol{D}$ liuchen715@tju.edu.cn )

Tianjin University

\section{Research Article}

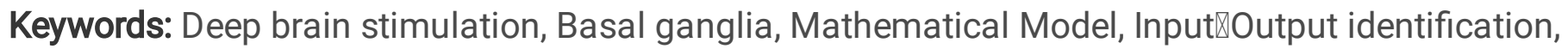
Generalized minimum variance algorithm

Posted Date: April 20th, 2021

DOI: https://doi.org/10.21203/rs.3.rs-431596/v1

License: (9) This work is licensed under a Creative Commons Attribution 4.0 International License. Read Full License 


\section{Adaptive Parameter Modulation of Deep Brain Stimulation in a Computational Model of Basal Ganglia-thalamic network}

Yulin Zhu ${ }^{\mathrm{a}}$, Jiang Wang ${ }^{\mathrm{a}}$, Siyuan Chang ${ }^{\mathrm{a}}$, Huiyan $\mathrm{Li}^{\mathrm{b}}$, Bin Deng ${ }^{\mathrm{a}}$, and Chen Liu ${ }^{\mathrm{a}}$

${ }^{a}$ School of Electrical and Information Engineering, Tianjin University, Tianjin 30072, China

${ }^{b}$ School of Automation and Electrical Engineering, Tianjin University of Technology and Education, Tianjin, China

e-mail:liuchen715@tju.edu.cn

Abstract

Deep brain stimulation (DBS) has proven to be an effective treatment for Parkinson's disease (PD). Adaptive control strategies offer the potential to improve efficacy, limit side effects and save battery consumption via reducing the total amount of stimulation delivered. However, the mechanisms underlying the beneficial effects of DBS for PD remain poorly understood and are still under debate, which has hindered the development of closed-loop DBS. And during the chronically implanted phase, adaptive DBS needs to be further improved to maintain its advantages. In the design of new adaptive DBS, more insights into inaccuracies when establishing mathematical basal ganglia model, unknown external disturbance signal and dynamics of focal area should be considered. A controlled auto-regressive moving average is used as the representative description of stimulus-response relationship based on recursive extended least square method, where stimulation signal is applied to subthalamic nucleus (STN) and the feedback signal is selected as local field potential signal from globus pallidus (GPi). The generalized minimum variance algorithm is used for online update of stimulation frequency and amplitude in a closed-loop manner. Simulation results illustrated the efficiency of the proposed closed-loop stimulation methods in disrupting the aberrant beta band oscillation and restore normal firing pattern when compared with the PD state. Robustness of the adaptive algorithm was further verified through dynamic change of illness state. 
Keywords: Deep brain stimulation; Basal ganglia; Mathematical Model; InputOutput identification; Generalized minimum variance algorithm

\section{Introduction}

Parkinson's disease (PD) is the second most common neurodegenerative disorder associated with the degeneration of dopaminergic neurons in the substantia nigra par compacta [1]-[6]. Up till now, more than ten million people are suffering from the motor symptoms such as bradykinesia, rigidity, rest tremor, and postural and gait impairment worldwide [7], [8]. Conventional deep brain stimulation (DBS) has significantly improved the treatment of PD through continuously delivering high frequency electrical stimulation to specific subcortical targets, including the subthalamic nucleus (STN) and the internal segment of the globus pallidus (GPi) [9]-[13]. However, its open-loop mode induces limitation on sideeffects, battery-consumption and parameter-programming, which presents demands for a more automated and intelligent approach [14]-[19]. In recent years, closed-loop stimulation that utilizing patients' clinical states as a guide to alter stimulation parameters as necessary has attracted more attention [20]-[27]. It provides an effective approach to optimize clinical benefits whilst minimizing side effects and battery depletion. A critical step in the development of such systems is the identification of signal features or "biomarkers" which have the potential to quantify clinical states [28], [29]. To date, local field potentials (LFPs) have become a commonly adopted feedback signal for closing the loop due to its correlation to patients' clinical states and the ease with which they can be acquired [26], [30]-[32]. Little et al. adopted an "on-off" pattern to deliver the stimulation signals, which indicates that the stimulator is on only when the detected LFPs beta amplitude exceeds certain threshold [22]. Although this adaptive DBS was shown to be at least as effective as conventional DBS, and stimulation time and side effects were reduced, but from a chronically implanted phase its stun effect will disappear. Thus, new adaptive DBS directs to modulate the stimulation parameters based on new algorithm is required. Proportional control strategy has also shown potential in computational studies but restricted due to the time-consuming parameter adjustment process [33]. While it largely depended on the properties of controlled brain network, which left still a long way from clinical implementation. However, the precise mathematical model of the 
Parkinsonian focal area could not be established [34]. Besides, individual variation and illness progress need further to be characterized to guarantee precise stimulus mode [35]-[37]. The above two points put forwards exacting requirements for the development of closed-loop strategy. To address the problem, a self-tuning controller, which combines the recursive method of parameter estimation and different types of control algorithms, is proposed to form a real-time computer control system, thereby realizing the adaptive adjustment of parameters (including frequency and amplitude) of DBS signal. Considering the inherent time delay between excitatory or inhibitory neurotransmitter transmission between populations within Basal GangliaThalamus (BG-Th) model, it is necessary to predict the control effect in advance of this time delay so as to avoid suboptimal control effect [38]-[40]. With the advantage of simple in algorithm and easy to implement, minimum variance control has become the foundation of other self-tuning control algorithms [41], which could be utilized in our work to develop a predictive closed-loop system. The rest of the paper is organized as follows. In Section II, models and methods are introduced. Firstly, introduction of the computational model and selection of feedback signal were given, so as to generate input-output data for the identification of the stimulus-response relationship. Then, the control framework was described in detail. Computer simulation results were presented in Section III. Finally, the discussion and conclusion were given in Section IV.

\section{Methods}

In this section, we started by describing a previously proposed mathematical model of Parkinsonian focal zone, which was adopted here as the data generator and stimulation testbed. Considering that Parkinsonian behaviors were related to rhythmic firing patterns with oscillations at beta band [29], the relationship between the applied stimulus signal and the LFPs output was established through real-time online identification, based on which the closed-loop controller was developed and designed for selection of optimal stimulation to improve Parkinsonian state as shown in Fig. 2. 


\subsection{Model description}

The biological plausible mathematical BG-Th model that presented by So et al. [42] was modified from classical Rubin-Terman model [43]. Compared with the original Rubin-Terman model, the change of ionic current types and the network topological structure prompted the firing properties better match experimental data. Therefore, it was an excellent platform to study effects of neural activation or silencing and to test the adaptive control algorithm in this paper.

The BG-Th network was formed by excitatory or inhibitory synaptic connections among four primary nuclei including STN, GPe, GPi and Th. Each STN neuron received inhibitory connections from two GPe neurons. Each GP (GPe and GPi) neuron received excitatory inputs from two STN neurons and inhibitory inputs from two GPe neurons. Each Th neuron received inhibitory input from one GPi neuron. All connections that following a structured and deterministic pattern were illustrated in Fig. 1. The number of cells in each nucleus was 10, which was proven to be sufficient to generate same trends of normal and Parkinsonian states when compared with larger number of cells in each population. The single compartment Hodgkin-Huxley type equations were used to describe firing dynamics of STN, GPe, GPi and Th

$$
\begin{aligned}
& C_{m} \frac{d v_{\mathrm{STN}}}{d t}=-I_{1}-I_{\mathrm{Na}}-I_{\mathrm{K}}-I_{\mathrm{T}}-I_{\mathrm{Ca}}-I_{\mathrm{AHP}}-I_{\mathrm{GPe} \rightarrow \mathrm{STN}}+I_{\mathrm{app} \_\mathrm{STN}}+\left(I_{D B S}\right) \\
& C_{m} \frac{d v_{\mathrm{GPe}}}{d t}=-I_{1}-I_{\mathrm{Na}}-I_{\mathrm{K}}-I_{\mathrm{T}}-I_{\mathrm{Ca}}-I_{\mathrm{AHP}}+I_{\mathrm{STN} \rightarrow \mathrm{GPe}}-I_{\mathrm{GPe} \rightarrow \mathrm{GPe}}+I_{\mathrm{app} \_\mathrm{GPe}} \\
& C_{m} \frac{d v_{\mathrm{GPi}}}{d t}=-I_{1}-I_{\mathrm{Na}}-I_{\mathrm{K}}-I_{\mathrm{T}}-I_{\mathrm{Ca}}-I_{\mathrm{AHP}}+I_{\mathrm{STN} \rightarrow \mathrm{GPi}}-I_{\mathrm{GPe} \rightarrow \mathrm{GPi}}+I_{\mathrm{app} \_\mathrm{GPi}} \\
& C_{m} \frac{d v_{\mathrm{Th}}}{d t}=-I_{1}-I_{\mathrm{Na}}-I_{\mathrm{K}}-I_{\mathrm{T}}-I_{\mathrm{GPi} \rightarrow \mathrm{Th}}+I_{\mathrm{C}}
\end{aligned}
$$

where $C_{m}=1 \mu \mathrm{F} / \mathrm{cm}^{2}$ was the membrane capacitance, $v_{i}(i \in \mathrm{STN}, \mathrm{GPe}, \mathrm{GPi})$ represented the membrane potential. On the right side of equation, $I_{1}, I_{\mathrm{Na}}, I_{\mathrm{K}}, I_{\mathrm{T}}, I_{\mathrm{Ca}}, I_{\mathrm{AHP}}$ were respectively the leak current, the sodium current, the potassium current, the low-threshold calcium current, the high-threshold calcium current and the after hyper polarization potassium current. $I_{a p p_{-} i}(i \in \mathrm{STN}, \mathrm{GPe}, \mathrm{GPi})$ represented constant positive bias current that input from unmodeled brain regions, such as striatum. The transition from normal state 
to Parkinsonian states was modeled by changing the value of $I_{a p p_{i} i} \cdot p d=0$ represented the normal state and $p d=1$ represented the PD state, thus we got the following relationship

$$
\left\{\begin{array}{l}
I_{a p p_{-} S T N}=33-10 * p d \\
I_{a p p_{-} G P e}=20-13 * p d \\
I_{a p p_{-} G P i}=21-6 * p d
\end{array}\right.
$$

Besides, $I_{\alpha \rightarrow \beta}(\alpha, \beta \in \mathrm{STN}, \mathrm{GPe}, \mathrm{GPi})$ described the synaptic connections from pre-synaptic nucleus $\alpha$ to post-synaptic nucleus $\beta$,

$$
I_{\alpha \rightarrow \beta}=g_{\alpha \rightarrow \beta}\left(v_{\beta}-E_{\alpha \rightarrow \beta}\right) \sum_{j} s_{\alpha}^{j},
$$

where $g_{\alpha \rightarrow \beta}$ and $E_{\alpha \rightarrow \beta}$ represented the maximal synaptic conductance and reversal potential respectively, and $\sum_{j} s_{\alpha}^{j}$ was the sum of synaptic variables. In addition to ionic currents and synaptic currents, for Th neurons, $I_{\mathrm{C}}$ denoted a series of excitatory current pulses from cortical neurons and followed a gamma distribution with the average rate of $14 \mathrm{~Hz}$ and the variation of 0.2 , the amplitude and pulse duration of which were set to $3.5 \mu \mathrm{A} / \mathrm{cm}^{2}$ and $5 \mathrm{~ms}$ respectively. Moreover, the implementation of DBS stimulus took the form as

$$
I_{\mathrm{DBS}}=A_{\mathrm{DBS}} H\left(\sin \frac{2 \pi t}{\rho_{\mathrm{DBS}}}\right) \cdot\left[1-H\left(\sin \left(2 \pi t+\delta_{\mathrm{DBS}}\right) / \rho_{\mathrm{DBS}}\right)\right]
$$

Conventional effective stimulus parameters were usually set to be $A_{\mathrm{DBS}}=300 \mu \mathrm{A} / \mathrm{cm}^{2}, \delta_{\mathrm{DBS}}=0.3 \mathrm{~ms}$ and $\rho_{\mathrm{DBS}}=7.69 \mathrm{~ms}(130 \mathrm{~Hz})$. While in this paper, values of DBS parameters were modulated based on real-time calculation from closed-loop controller.

For the case of frequency modulation,

$$
\rho_{\mathrm{DBS}}=1000 / u(k)\left(f_{\min } \leq u(k) \leq f_{\max }\right)
$$

For the case of amplitude modulation,

$$
A_{\mathrm{DBS}}=u(k)\left(A_{\min } \leq u(k) \leq A_{\max }\right)
$$


where $f_{\max }$ and $f_{\min }$ were the maximum and minimum bounds of the modulated DBS frequency, $A_{\max }$ and $A_{\min }$ were the maximum and minimum bounds of the modulated DBS amplitude, and calculation of $u(k)$ was introduced in the following part. Details of equations and parameters were given in Table 1, where membrane potentials, synaptic conductance, currents were expressed in $m V$, $\mathrm{mS} / \mathrm{cm}^{2}$ and $\mu \mathrm{A} / \mathrm{cm}^{2}$ respectively. Simulations were implemented in Matlab R2019a (The Math Works, Natick, MA), and the equations were solved using the forward Euler method with a time step of $0.01 \mathrm{~ms}$.

\subsection{Identification of the stimulus-response relationship}

PD was a progressive neurodegenerative disease. Selection of appropriate biomarker to capture ongoing clinical changes was a critical process. It has been proved that beta-band oscillation of LFPs signals was related to motor symptoms of bradykinesia and rigidity across patients. Recent research presented the evidence that LFPs signals that directly recorded from DBS electrodes appear to be a promising source of feedback signal. Based on the mathematical BG-Th network, the LFPs signal of GPi nucleus [44] could be computed by

$$
\mathrm{LFP}_{\mathrm{GPi}}=I_{\mathrm{STN} \rightarrow \mathrm{GPi}}-I_{\mathrm{GPe} \rightarrow \mathrm{GPi}}+I_{\text {app_GPi }} \text {, }
$$

which was approximated by the sum of transmembrane currents.

However, due to the characteristic of highly nonlinearity within BG-Th network, it presented huge challenges in the design of closed-loop control algorithm. A recursive extended least square (RELS) method was proposed to realize online real-time parameter estimation, therein the model structure could be delineated by the controlled auto-regressive moving average (CARMA) model

$$
A\left(z^{-1}\right) y(k)=z^{-d} B\left(z^{-1}\right) u(k)+C\left(z^{-1}\right) \xi(k)
$$

where $u(k)$ represented the input DBS stimulus signal (to STN), $y(k)$ represented the output LFPs signal (from GPi) at time $k T_{s} \quad\left(T_{s}=0.01 \mathrm{~ms}\right.$ was the sampling interval), $\xi(k)$ was Gaussian white noise series that representing the sum of the stochastic perturbation and unmodeled dynamics. and $d$ was the time delay. Coefficients of polynomial 


$$
\left\{\begin{array}{l}
A\left(z^{-1}\right)=1+a_{1} z^{-1}+a_{2} z^{-2}+\ldots+a_{n_{a}} z^{-n_{a}} \\
B\left(z^{-1}\right)=b_{0}+b_{1} z^{-1}+b_{2} z^{-2}+\ldots+b_{n_{b}} z^{-n_{b}} \\
C\left(z^{-1}\right)=1+c_{1} z^{-1}+c_{2} z^{-2}+\ldots+c_{n_{c}} z^{-n_{c}}
\end{array}\right.
$$

were to be determined by detectable input and output data.

Firstly, the CARMA model was written in least squares form

$$
y(k)=\boldsymbol{\varphi}^{T}(k) \boldsymbol{\theta}+\xi(k)(10)
$$

where

$$
\left\{\begin{array}{c}
\boldsymbol{\varphi}(k)=\left[-y(k-1), \ldots,-y\left(k-n_{a}\right), u(k-d),\right. \\
\left.\ldots, u\left(k-d-n_{b}\right), \xi(k-1), \ldots, \xi\left(k-n_{c}\right)\right]^{T} \\
\theta=\left[a_{1}, \ldots, a_{n_{a}}, b_{0}, \ldots, b_{n_{b}}, c_{1}, \ldots, c_{n_{c}}\right] .
\end{array}\right.
$$

Due to the uncertainty of $\xi(k)$ in $\varphi(k)$, it was substituted using the estimation value $\hat{\xi}(k)=y(k)-\hat{y}(k)=y(k)-\widehat{\boldsymbol{\varphi}}^{T}(k) \boldsymbol{\theta}$. Then, least squares estimation was performed to minimize the performance evaluation

$$
J=\sum \varepsilon^{2}(k)=\sum\left[y(k)-\widehat{\boldsymbol{\varphi}}^{T}(k) \boldsymbol{\theta}\right]^{2}
$$

If we took the derivative of $J$ with respect to $\hat{\boldsymbol{\theta}}$ (let it equal to 0 ), then continuously revised the latest estimation parameter $\widehat{\boldsymbol{\theta}}(k)$ based on last $\widehat{\boldsymbol{\theta}}(k-1)$ and correction term, it could be derived that

$$
\left\{\begin{array}{l}
\widehat{\boldsymbol{\theta}}(k)=\hat{\boldsymbol{\theta}}(k-1)+\boldsymbol{K}(k)\left[y(k)-\hat{\boldsymbol{\varphi}}^{T}(k) \boldsymbol{\theta}(k-1)\right] \\
\boldsymbol{K}(k)=\frac{\boldsymbol{P}(k-1) \hat{\boldsymbol{\varphi}}(k)}{1+\widehat{\boldsymbol{\varphi}}^{T}(k) \boldsymbol{P}(k-1) \hat{\boldsymbol{\varphi}}(k)} \\
\boldsymbol{P}(k)=\left[\boldsymbol{I}-\boldsymbol{K}(k) \hat{\boldsymbol{\varphi}}^{T}(k)\right] \boldsymbol{P}(k-1)
\end{array}\right.
$$

\subsection{Controller design}

To automatically adjust DBS parameters, a real-time computer control system was established by combing the recursive method of parameter estimation and selftuning control method. Considering the existence of time lag between DBS action and LFPs output, the generalized minimum variance control (GMVC) predicted the output in advance, and then designed the control law to guarantee the stability of BG-Th network [45].

According to the CARMA model of Eq. (10), 


$$
y(k+d)=\frac{B\left(z^{-1}\right)}{A\left(z^{-1}\right)} u(k)+\frac{C\left(z^{-1}\right)}{A\left(z^{-1}\right)} \xi(k+d)
$$

since that $\xi(k+1), \ldots, \xi(k+d)$ could not be acquired at time $k T_{s}, \frac{C\left(z^{-1}\right)}{A\left(z^{-1}\right)}$ was divided into two parts based on observability and unobservability

$$
\left\{\begin{array}{l}
F\left(z^{-1}\right)=1+f_{1} z^{-1}+\ldots+f_{d-1} z^{-(d-1)} \\
G\left(z^{-1}\right)=g_{0}+g_{1} z^{-1}+\ldots+g_{n_{a}-1} z^{-\left(n_{a}-1\right)}
\end{array}\right.
$$

Therefore,

$$
\frac{C\left(z^{-1}\right)}{A\left(z^{-1}\right)}=F\left(z^{-1}\right)+\frac{z^{-d} G\left(z^{-1}\right)}{A\left(z^{-1}\right)}
$$

Combined with $\xi(k)=\frac{A\left(z^{-1}\right)}{C\left(z^{-1}\right)} y(k)-\frac{B\left(z^{-1}\right)}{C\left(z^{-1}\right)} z^{-d} u(k)$, it could be obtained that

$$
y(k+d)=F\left(z^{-1}\right) \xi(k+d)+\frac{B\left(z^{-1}\right) F\left(z^{-1}\right)}{C\left(z^{-1}\right)} u(k)+\frac{G\left(z^{-1}\right)}{C\left(z^{-1}\right)} y(k)
$$

In view that $\xi(k+d)$ was independent of $\{y(k), y(k-1), \ldots, u(k), u(k-1), \ldots\}$, the optimal least variance prediction was

$$
\begin{gathered}
y^{*}(k+d)=\widehat{y}(k+d)=\frac{B\left(z^{-1}\right) F\left(z^{-1}\right)}{C\left(z^{-1}\right)} u(k)+\frac{G\left(z^{-1}\right)}{C\left(z^{-1}\right)} y(k) \\
y(k+d)=F\left(z^{-1}\right) \xi(k+d)+y^{*}(k+d)
\end{gathered}
$$

Further, to improve closed-loop system performance, to soften the desired output and to constraint on control input, weighted polynomial $P\left(z^{-1}\right), R\left(z^{-1}\right)$ and $Q\left(z^{-1}\right)$ were introduced

$$
\left\{\begin{array}{l}
P\left(z^{-1}\right)=1+p_{1} z^{-1}+p_{2} z^{-2}+\ldots+p_{n_{p}} z^{-n_{p}} \\
R\left(z^{-1}\right)=1+r_{1} z^{-1}+r_{2} z^{-2}+\ldots+r_{n_{r}} z^{-n_{r}} \\
Q\left(z^{-1}\right)=1+q_{1} z^{-1}+q_{2} z^{-2}+\ldots+q_{n_{q}} z^{-n_{q}}
\end{array}\right.
$$

By this time, the cost function was expressed in 


$$
\begin{aligned}
C & =E\left\{\left[P\left(z^{-1}\right) y(k+d)-R\left(z^{-1}\right) y_{r}(k+d)\right]^{2}\right\}+\left[Q\left(z^{-1}\right) u(k)\right]^{2} \\
& =E\left\{\left[P F \xi(k+d)+P y^{*}(k+d)-R y_{r}(k+d)\right]^{2}\right\}+[Q u(k)]^{2}
\end{aligned}
$$

The first term was omitted due to its independence with past input and output, then we took the partial derivative of rest terms in Eq. (21) with respect to $u(k)$

$$
2\left[P y^{*}(k+d)-R y_{r}(k+d)\right] \frac{\partial P y^{*}(k+d)}{\partial u(k)}+2[Q u(k)] \frac{\partial Q u(k)}{\partial u(k)}=0
$$

Substituting $\frac{\partial P y^{*}(k+d)}{\partial u(k)}=b_{0}, \frac{\partial Q u(k)}{\partial u(k)}=q_{0}$ into Eq. (22),

$$
\left[P y^{*}(k+d)-R y_{r}(k+d)\right] * b_{0}+Q u(k)^{*} q_{0}=0
$$

the generalized minimum variance control law was obtained as

$$
u(k)=\frac{R y_{r}(k+d)-G P y(k)}{\frac{q_{0}}{b_{0}} C Q+B F P}
$$

where the control signal at time $k T_{s}$ was obtained to be applied to the BG-Th model target.

\section{Results}

\subsection{Simulated BG-Th network firing pattern}

The transition from normal state to Parkinsonian state was achieved by changing the level of bias currents to STN, GPe and GPi neurons. As shown in Fig. 3 (a), (b) and (c), the STN, GPe and GPi neurons exhibited regular spiking in the normal physiological condition. While in the PD state, a rhythmic bursting fashion occurred, especially in the GPe and GPi neurons as shown in Fig. 3 (d), (e) and (f). Then, we measured the relay ability of Th neurons in the reference of cortex input. Error index

$$
E I=\frac{N_{\text {error }}}{N_{c}}
$$

was defined as a valid proxy to quantify the reliability of Th neurons. When the transmembrane voltage crossed the threshold of $-40 \mathrm{mV}$, a spike of Th neuron was 
detected. Correspondingly, an error was defined when a spike failure occurred in response to cortex input. $N_{\text {error }}$ represented the total number of errors (including missing spikes, rebound bursts and spurious events) in thalamic transmission, and $N_{c}$ summed the number of cortex input pulses. As shown in Fig. 4, more firing errors occured due to rhythmic pattern of GPi neurons in the pathological condition, with the error index $E I$ increased from 0.0073 (normal condition) to 0.4365 (PD condition). Besides, GPi LFPs that approximated by the sum of transmembrane currents were presented in Fig. 5, power spectral analysis of which manifested a beta-band oscillation under PD condition, when compared with normal condition.

\subsection{Performance of stimulus-response identification}

A CARMA model was identified to establish the relationship between DBS stimulation signal and the LFPs response signal. Based on the obtained input and output data from the BG-Th network, it was necessary to select appropriate order parameters by quantifying the estimation accuracy. Since parameters $\left[c_{1}, c_{2}, \ldots, c_{n_{c}}\right]$ were used to measure the influence of uncertain dynamics, $n_{c}$ was set to 3 . The root mean square error

$$
e=\sqrt{\frac{1}{N} \sum_{n=1}^{k}(y(k)-\widehat{y}(k))^{2}}
$$

between actual value and estimated value of LFPs signal in GPi was calculated and compared among different model structures. As shown in Fig. 6 (a), it was found that identified error was always smaller than 0.95 and simultaneously it decreased with the increase of $n_{a}$ and $n_{b}$. But to reduce computational complexity $n_{a}$ and $n_{b}$ were all set to 4 . In this case, evolution of estimation results was illustrated in Fig. 6 (b), where all identified parameters rapidly converged to stable values. Correspondingly, the identification performance was exhibited in Fig. 6 (c), where the red solid line displayed the original data that recorded from BG-Th network output and the blue dotted line represented the estimated data generated from CARMA model. The result suggested that the CARMA model provided a good fit to the obtained input-output data. 


\subsection{Efficacy of GMVC strategy}

The design of closed-loop DBS system was based on the identified parameters of the CARMA model, with the frequency or amplitude calculated based on the adaptive generalized minimum variance controller. For the case when DBS was applied to STN neurons with real-time frequency modulation since $\mathrm{t}=1000 \mathrm{~ms}$, rhythmic bursting firing pattern of GPi was obviously suppressed as denoted by comparison of LFPs waveform signal before and after stimulation as shown in Fig. 7 (a). Fig. 7 (b) indicated the change of stimulation frequency by the sparse and intensive distribution stimulus pulse, where and $f_{\max }$ and $f_{\min }$ were set to $200 \mathrm{~Hz}$ and $50 \mathrm{~Hz}$ to simulate clinically effective frequency bands. As shown in Fig. 7 (c), quantitative analysis through power spectrum indicated the disrupted pathological frequency structure after closed-loop DBS amplitude modulation. Similarly, for the case when DBS was applied to STN neurons with real-time amplitude modulation, suppression of rhythmic oscillated firing pattern and disruption of pathological spectrum structure were realized since $t=1000 \mathrm{~ms}$ as shown in Fig. 8.

Further analysis of closed-loop performance was developed in terms of interspike interval (ISI) changes as shown in Fig. 9, where abscissa presented ISI distribution and ordinate empirical cumulative distribution function. Regular spike firing in normal condition was always accompanied by uniform distribution of small ISI, while burst firing pattern was accompanied by dispersion of ISI. Specifiaclly, as shown in Fig. 9, ISI distributed mainly around $20 \mathrm{~ms}$ in normal state. In PD state, distribution of ISI focused around $10 \mathrm{~ms}$ and $60 \mathrm{~ms}$, which represented the strong polarization that induced by increase of pathological burst firing. As the stimulus signal was applied, the pathological condition of ISI distribution was gradually recovered with the tendency towards normal distribution, under both closed-loop frequency modulation and amplitude modulation.

As described ahead, the Parkinsonian state was simulated by decreasing the constant bias currents that applied to the STN, GPe and GPi neurons. Directly switching $I_{a p p_{-} i}$ from a higher value to a lower value apparently ignored the possible intermediate states resulting from diverse courses of disease caused by individual differences, thus we defined a dynamic parameter $p d$ to quantify 
disease severity. Correspondingly, the robustness and adaptability of GMVC strategy on closed-loop control performance was tested by continuously changing $p d$ from 0 to 1 . Wherein, the approximate integration of spectral power corresponding to $12-35 \mathrm{~Hz}$ was calculated as a Beta index to distinguish normal state from PD state. As shown in Fig. 10, the red error bar presented the trend of Beta index with the increase of $p d$. Real-time closed-loop frequency modulation and amplitude modulation was implemented as $p d$ increased from 0.1 to 1 . In both cases, the increased Beta index was almost pulled back to normal state as denoted by grey error bar with yellow round markers and grey error bar with green square markers, indicating the restoration of the spectral features.

\section{Discussion and conclusion}

The objective of this study was to design a closed-loop control strategy to suppress Parkinsonian state. To advance the progress of neurophysiological experiments, reveal the principles of information processing and various functions of the nervous system, we focused on the model research to inspire new treatments for neurological disorders. In this paper, the computational BG-Th network that consisting of STN, GPe, GPi and Th neurons was constructed based on Hodgkin-Huxley type equation, where parameter settings and synaptic connections were formed obeying anatomy and physiological properties. Based on the findings that the rhythmically oscillatory activity of LFPs is a remarkable PDrelated feature, LFPs of GPi neurons was considered as a feedback signal that distinguish normal condition from PD condition. Due to the lack of adequate physiological details and uncertainties in noise or disturbances, a black-box model approach based on the CARMA model was utilized to remedy above limitation, where the mathematical model generated data was input to the black box. Therefore, it was expected to describe the relationship between stimulation signal and neuronal response according to the identification method. Then adaptive reprogramming DBS stimulation parameters in reducing the adverse effects of continuous high-frequency DBS was tested through the proposed self-tuning control strategy GMVC.

Numerical simulation results illustrated the possibility of mitigating intractable Parkinsonism by GMVC, including the suppression of abnormally enhanced beta band oscillation and the regulation of interspike interval distribution. Firstly, the 
intrinsic transmission time delay between STN and GPi cannot be neglected, which was considered in the design of the closed-loop system. Secondly, to overcome model limitation caused by symptom severity, it was hypothesized to represent possible dynamics by adjusting the parameter $p d$ from 0 to 1 , and the GMVC strategy reached remarkably excellent controlling performance. Although robustness of the proposed control algorithm was tested based on data extracted from a modified computational model from Rubin-Terman model, it was still far from enough to reveal the real brain network and inevitably ignore considerable physiological knowledge. Therefore, the potential application of GMVC in future clinical DBS will consider much more issues, such as which is the most appropriate biomarker to be the feedback variable and how they vary across patients and time, which of or what combination of the parameters (amplitude, frequency, or pulse width) is the best beneficial. Further verification of this method in other computational models and animal experiments was expected in future work.

Acknowledgements

This work was supported by the Natural Science Foundation of Tianjin, China (Grant Nos. 20JCQNJC01160 and 18JCZDJC32000) and the Foundation of Tianjin University under Grant 2020XRG-0018. The authors also gratefully acknowledge the financial support provided by Opening Fundation of Key Laboratory of Opto-technology and Intelligent Control (Lanzhou Jiaotong University), Ministry of Education (KFKT2020-01).

\section{Conflict of Interest}

We declare that we have no financial and personal relationships with other people or organizations that can inappropriately influence our work, there is no professional or other personal interest of any nature or kind in any product, service and/or company that could be construed as influencing the position presented in.

References

1. Cutler, R.L., Fernandez-Llimos, F., Frommer, M., Benrimoj, C., Garcia-Cardenas, V.: Economic impact of medication non-adherence by disease groups: a systematic review. BMJ Open. 8, e016982 (2018). https://doi.org/10.1136/bmjopen-2017-016982 
2. Kimpara, T., Takeda, A.: [Parkinson's Disease in the Oldest-Old]. Brain and nerve $=$ Shinkei kenkyu no shinpo. 72, 1345-1352 (2020). https://doi.org/10.11477/mf.1416201691

3. Marras, C., Beck, J.C., Bower, J.H., Roberts, E., Ritz, B., Ross, G.W., Abbott, R.D., Savica, R., Van Den Eeden, S.K., Willis, A.W., Tanner, C.M.: Prevalence of Parkinson's disease across North America. npj Parkinsons Dis. 4, 21 (2018). https://doi.org/10.1038/s41531-018$\underline{0058-0}$

4. Fan, K.Y., Baufreton, J., Surmeier, D.J., Chan, C.S., Bevan, M.D.: Proliferation of External Globus Pallidus-Subthalamic Nucleus Synapses following Degeneration of Midbrain Dopamine Neurons. Journal of Neuroscience. 32, 13718-13728 (2012). https://doi.org/10.1523/JNEUROSCI.5750-11.2012

5. Albin, R.L., Young, A.B., Penney, J.B.: The functional anatomy of basal ganglia disorders. Trends in neurosciences. 12, 366-75 (1989). https://doi.org/10.1016/0166-2236(89)90074-X

6. Yu, Y., Wang, X., Wang, Q., Wang, Q.: A review of computational modeling and deep brain stimulation: applications to Parkinson's disease. Applied Mathematics and MechanicsEnglish Edition. 41, 1747-1768 (2020). https://doi.org/10.1007/s10483-020-2689-9

7. Jankovic, J.: Parkinson's disease: clinical features and diagnosis. J. Neurol. Neurosurg. Psychiatry. 79, 368-376 (2008). https://doi.org/10.1136/jnnp.2007.131045

8. Dexter, D.T., Jenner, P.: Parkinson disease: from pathology to molecular disease mechanisms. Free Radic. Biol. Med. 62, 132-144 (2013). https://doi.org/10.1016/j.freeradbiomed.2013.01.018

9. Rodriguez-Oroz, M.C., Obeso, J.A., Lang, A.E., Houeto, J.L., Pollak, P., Rehncrona, S., Kulisevsky, J., Albanese, A., Volkmann, J., Hariz, M.I., Quinn, N.P., Speelman, J.D., Guridi, J., Zamarbide, I., Gironell, A., Molet, J., Pascual-Sedano, B., Pidoux, B., Bonnet, A.M., Agid, Y., Xie, J., Benabid, A.L., Lozano, A.M., Saint-Cyr, J., Romito, L., Contarino, M.F., Scerrati, M., Fraix, V., Van Blercom, N.: Bilateral deep brain stimulation in Parkinson's disease: a multicentre study with 4 years follow-up. Brain. 128, 2240-2249 (2005). https://doi.org/10.1093/brain/awh571

10. Benabid, A.L.: Deep brain stimulation for Parkinson's disease. Curr. Opin. Neurobiol. 13, 696-706 (2003). https://doi.org/10.1016/j.conb.2003.11.001

11. Sidiropoulos, C., LeWitt, P.A.: Gpi Vs Stn Deep Brain Stimulation for Parkinson Disease: Three-Year Follow-Up. Neurology. 87, 745-745 (2016

12. Jakobs, M., Lee, D.J., Lozano, A.M.: Modifying the progression of Alzheimer's and Parkinson's disease with deep brain stimulation. Neuropharmacology. 171, 107860 (2020). https://doi.org/10.1016/j.neuropharm.2019.107860

13. Assessment of the effects of subthalamic stimulation in Parkinson disease patients by artificial neural network - IEEE Conference Publication, https://ieeexplore.ieee.org/document/5333545

14. Okun, M.S., Foote, K.D.: Parkinson's disease DBS: what, when, who and why? The time has come to tailor DBS targets. Expert Review of Neurotherapeutics. 10, 1847-1857 (2010). https://doi.org/10.1586/ERN.10.156 
15. Cyron, D.: Mental Side Effects of Deep Brain Stimulation (DBS) for Movement Disorders: The Futility of Denial. Frontiers in Integrative Neuroscience. 10, 17 (2016). https://doi.org/10.3389/foint.2016.00017

16. Mohammed, A., Bayford, R., Demosthenous, A.: A Framework for Adapting Deep Brain Stimulation Using Parkinsonian State Estimates. Front. Neurosci. 14, 499 (2020). https://doi.org/10.3389/fnins.2020.00499

17. Arlotti, M., Rosa, M., Marceglia, S., Barbieri, S., Priori, A.: The adaptive deep brain stimulation challenge. Parkinsonism \& Related Disorders. 28, 12-17 (2016). https://doi.org/10.1016/j.parkreldis.2016.03.020

18. Picillo, M., Lozano, A.M., Kou, N., Munhoz, R.P., Fasano, A.: Programming Deep Brain Stimulation for Parkinson's Disease: The Toronto Western Hospital Algorithms. Brain Stimulation. 9, 425-437 (2016). https://doi.org/10.1016/j.brs.2016.02.004

19. Wang, S., Na, J.: Parameter Estimation and Adaptive Control for Servo Mechanisms With Friction Compensation. IEEE Transactions on Industrial Informatics. 16, 6816-6825 (2020). https://doi.org/10.1109/TII.2020.2971056

20. Graupe, D., Basu, I., Tuninetti, D., Vannemreddy, P., Slavin, K.V.: Adaptively controlling deep brain stimulation in essential tremor patient via surface electromyography. Neurological Research. 32, 899-904 (2010). https://doi.org/10.1179/016164110X12767786356354

21. Rosin, B., Slovik, M., Mitelman, R., Rivlin-Etzion, M., Haber, S.N., Israel, Z., Vaadia, E., Bergman, H.: Closed-Loop Deep Brain Stimulation Is Superior in Ameliorating Parkinsonism. Neuron. 72, 370-384 (2011). https://doi.org/10.1016/j.neuron.2011.08.023

22. Little, S., Pogosyan, A., Neal, S., Zavala, B., Zrinzo, L., Hariz, M., Foltynie, T., Limousin, P., Ashkan, K., FitzGerald, J., Green, A.L., Aziz, T.Z., Brown, P.: Adaptive Deep Brain Stimulation in Advanced Parkinson Disease. Ann. Neurol. 74, 449-457 (2013). https://doi.org/10.1002/ana.23951

23. Basu, I., Graupe, D., Tuninetti, D., Shukla, P., Slavin, K.V., Metman, L.V., Corcos, D.M.: Pathological tremor prediction using surface electromyogram and acceleration: potential use in "ON-OFF" demand driven deep brain stimulator design. Journal of Neural Engineering. 10, 036019 (2013). https://doi.org/10.1088/1741-2560/10/3/036019

24. Yamamoto, T., Katayama, Y., Ushiba, J., Yoshino, H., Obuchi, T., Kobayashi, K., Oshima, H., Fukaya, C.: On-Demand Control System for Deep Brain Stimulation for Treatment of Intention Tremor. Neuromodulation. 16, 230-235 (2013). https://doi.org/10.1111/j.1525$\underline{1403.2012 .00521 . x}$

25. Hosain, M.K., Kouzani, A., Tye, S.: Closed loop deep brain stimulation: an evolving technology. Australasian Physical \& Engineering Sciences in Medicine. 37, 619-634 (2014). https://doi.org/10.1007/s13246-014-0297-2

26. Rosa, M., Arlotti, M., Ardolino, G., Cogiamanian, F., Marceglia, S., Di Fonzo, A., Cortese, F., Rampini, P.M., Priori, A.: Adaptive Deep Brain Stimulation in a Freely Moving Parkinsonian Patient. Mov. Disord. 30, 1003-1005 (2015). https://doi.org/10.1002/mds.26241 
27. Tinkhauser, G., Pogosyan, A., Little, S., Beudel, M., Herz, D.M., Tan, H., Brown, P.: The modulatory effect of adaptive deep brain stimulation on beta bursts in Parkinson's disease. Brain. 140, 1053-1067 (2017). https://doi.org/10.1093/brain/awx010

28. Parastarfeizabadi, M., Kouzani, A.Z.: Advances in closed-loop deep brain stimulation devices. J. NeuroEng. Rehabil. 14, 79 (2017). https://doi.org/10.1186/s12984-017-0295-1

29. Hoang, K.B., Cassar, I.R., Grill, W.M., Turner, D.A.: Biomarkers and Stimulation Algorithms for Adaptive Brain Stimulation. Frontiers in Neuroscience. 11, 564 (2017). https://doi.org/10.3389/fnins.2017.00564

30. Arlotti, M., Marceglia, S., Foffani, G., Volkmann, J., Lozano, A.M., Moro, E., Cogiamanian, F., Prenassi, M., Bocci, T., Cortese, F., Rampini, P., Barbieri, S., Priori, A.: Eight-hours adaptive deep brain stimulation in patients with Parkinson disease. Neurology. 90, E971-506 (2018). https://doi.org/10.1212/WNL.0000000000005121

31. Velisar, A., Syrkin-Nikolau, J., Blumenfeld, Z., Trager, M.H., Afzal, M.F., Prabhakar, V., Bronte-Stewart, H.: Dual threshold neural closed loop deep brain stimulation in Parkinson disease patients. Brain Stimul. 12, 868-876 (2019). https://doi.org/10.1016/j.brs.2019.02.020

32. Priori, A., Foffani, G., Rossi, L., Marceglia, S.: Adaptive deep brain stimulation (aDBS) controlled by local field potential oscillations. Experimental Neurology. 245, 77-86 (2013). https://doi.org/10.1016/j.expneurol.2012.09.013

33. Gorzelic, P., Schiff, S.J., Sinha, A.: Model-based rational feedback controller design for closed-loop deep brain stimulation of Parkinson's disease. Journal of Neural Engineering. 10, 026016 (2013). https://doi.org/10.1088/1741-2560/10/2/026016

34. Curchoe, C.L.: All Models Are Wrong, but Some Are Useful. Journal of Assisted Reproduction and Genetics. 37, 2389-2391 (2020). https://doi.org/10.1007/s10815-02001895-3

35. Johnson, L.A., Nebeck, S.D., Muralidharan, A., Johnson, M.D., Baker, K.B., Vitek, J.L.: Closed-Loop Deep Brain Stimulation Effects on Parkinsonian Motor Symptoms in a NonHuman Primate - Is Beta Enough? Brain Stimulation. 9, 892-896 (2016). https://doi.org/10.1016/j.brs.2016.06.051

36. Fleming, J.E., Orlowski, J., Lowery, M.M., Chaillet, A.: Self-Tuning Deep Brain Stimulation Controller for Suppression of Beta Oscillations: Analytical Derivation and Numerical Validation. Frontiers in neuroscience. 14, 639-639 (2020). https://doi.org/10.3389/fnins.2020.00639

37. Su, F., Kumaravelu, K., Wang, J., Grill, W.M.: Model-Based Evaluation of Closed-Loop Deep Brain Stimulation Controller to Adapt to Dynamic Changes in Reference Signal. Front. Neurosci. 13, 956 (2019). https://doi.org/10.3389/fnins.2019.00956

38. Gillies, A., Willshaw, D.: Membrane channel interactions underlying rat subthalamic projection neuron rhythmic and bursting activity. J. Neurophysiol. 95, 2352-2365 (2006). https://doi.org/10.1152/jn.00525.2005

39. Kita, H., Kitai, S.T.: Intracellular study of rat globus pallidus neurons: membrane properties and responses to neostriatal, subthalamic and nigral stimulation. Brain research. 564, 296305 (1991). https://doi.org/10.1016/0006-8993(91)91466-E 
40. Kumaravelu, K., Brocker, D.T., Grill, W.M.: A biophysical model of the cortex-basal ganglia-thalamus network in the 6-OHDA lesioned rat model of Parkinson's disease. J Comput Neurosci. 40, 207-229 (2016). https://doi.org/10.1007/s10827-016-0593-9

41. Multi-variable Generalized Minimum Variance Control with Time-delay Using Interactor Matrix - IEEE Conference Publication, https://ieeexplore.ieee.org/document/8861635

42. So, R.Q., Kent, A.R., Grill, W.M.: Relative contributions of local cell and passing fiber activation and silencing to changes in thalamic fidelity during deep brain stimulation and lesioning: a computational modeling study. Journal of Computational Neuroscience. 32, 499519 (2012). https://doi.org/10.1007/s10827-011-0366-4

43. Rubin, J.E., Terman, D.: High frequency stimulation of the subthalamic nucleus eliminates pathological thalamic rhythmicity in a computational model. J. Comput. Neurosci. 16, 211235 (2004). https://doi.org/10.1023/B:JCNS.0000025686.47117.67

44. Mazzoni, A., Lindén, H., Cuntz, H., Lansner, A., Panzeri, S., Einevoll, G.T.: Computing the Local Field Potential (LFP) from Integrate-and-Fire Network Models. PLoS Comput Biol. 11, e1004584 (2015). https://doi.org/10.1371/journal.pcbi.1004584

45. Qin, Z.: A Generalized Minimum Variance Self-Tuning Controller with Pole-Assignment. IFAC Proceedings Volumes. 18, 403-408 (1985). https://doi.org/10.1016/S1474$\underline{6670(17) 60593-7}$

46. Duchet, B., Ghezzi, F., Weerasinghe, G., Tinkhauser, G., Kühn, A.A., Brown, P., Bick, C., Bogacz, R.: Average beta burst duration profiles provide a signature of dynamical changes between the ON and OFF medication states in Parkinson's disease. Neuroscience (2020) 


\section{Figure Legends}

(a)

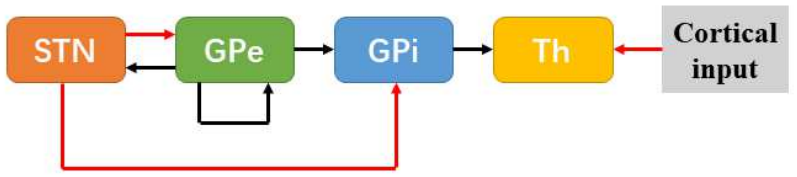

(b)

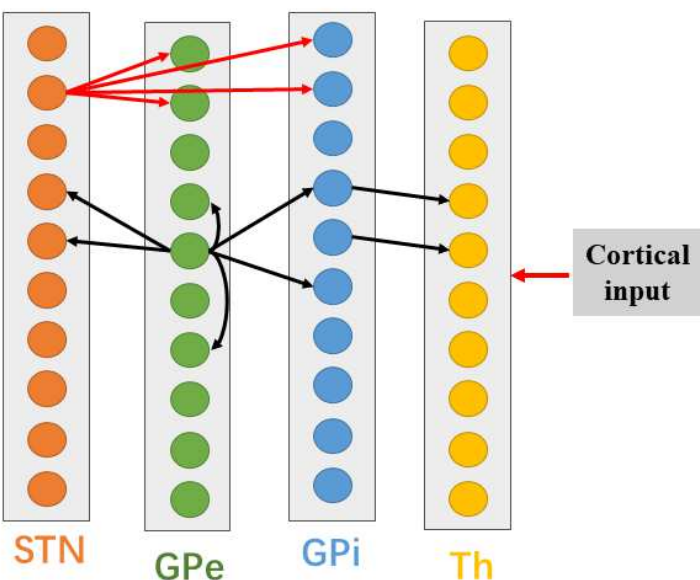

Fig. 1. BG-Th network model. (a) indicated the network layout and (b) depicted details of synaptic connections among STN, GPe, GPi and Th nucleus within the network, where red arrows denote excitatory connections andblack arrows denote inhibitory connections.

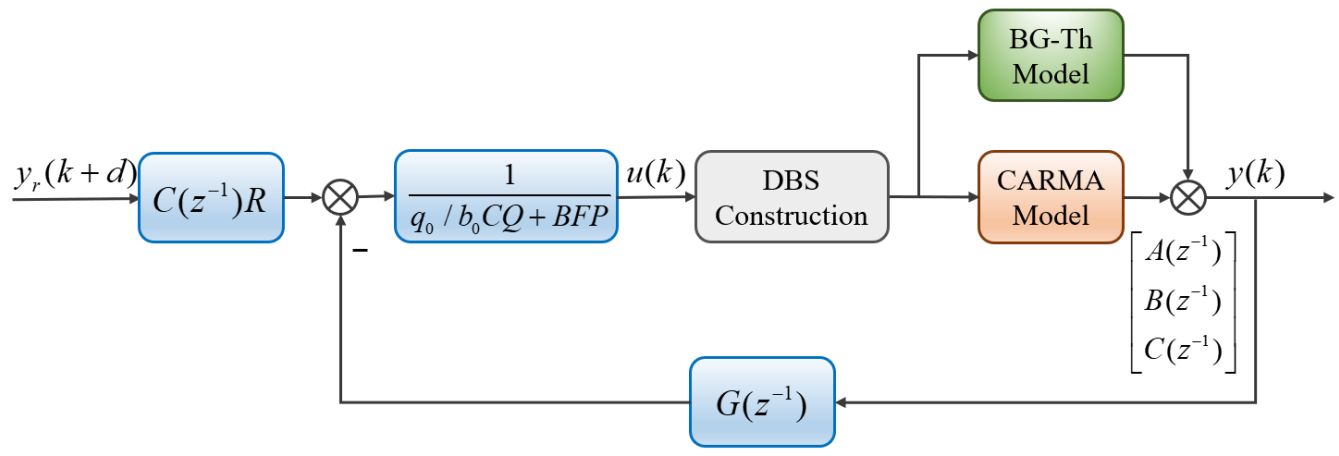

Fig.2 The diagram of CARMA model-based generalized minimum variance control system 

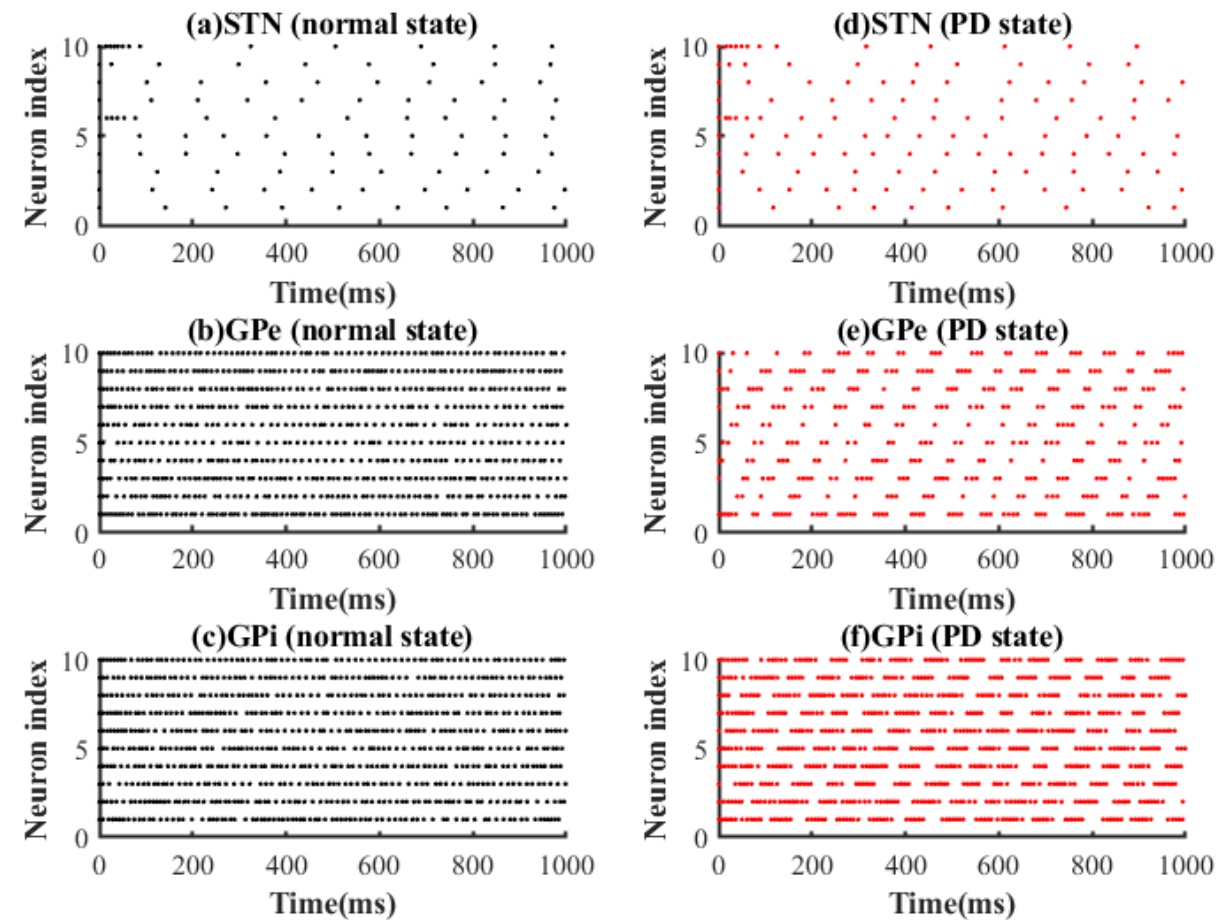

Fig. 3 Firing rastergrams of STN, GPe and GPi neurons under normal ((a), (b) and (c)) condition and PD ((d), (e) and (f)) condition.

(a)

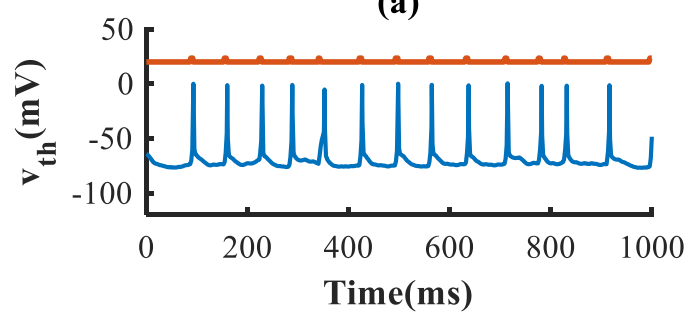

(b)

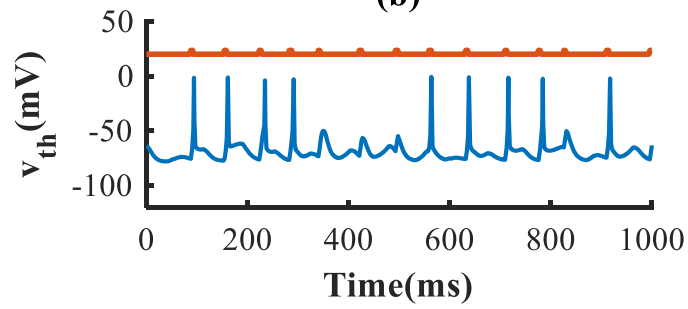

Fig. 4. Membrane potential traces of the Th neurons. Orange pulse trains denoted excitatory cortex input signals, blue traces in upper and bottom pannels represented the normal condition (a) and the PD condition (b), respectively. 

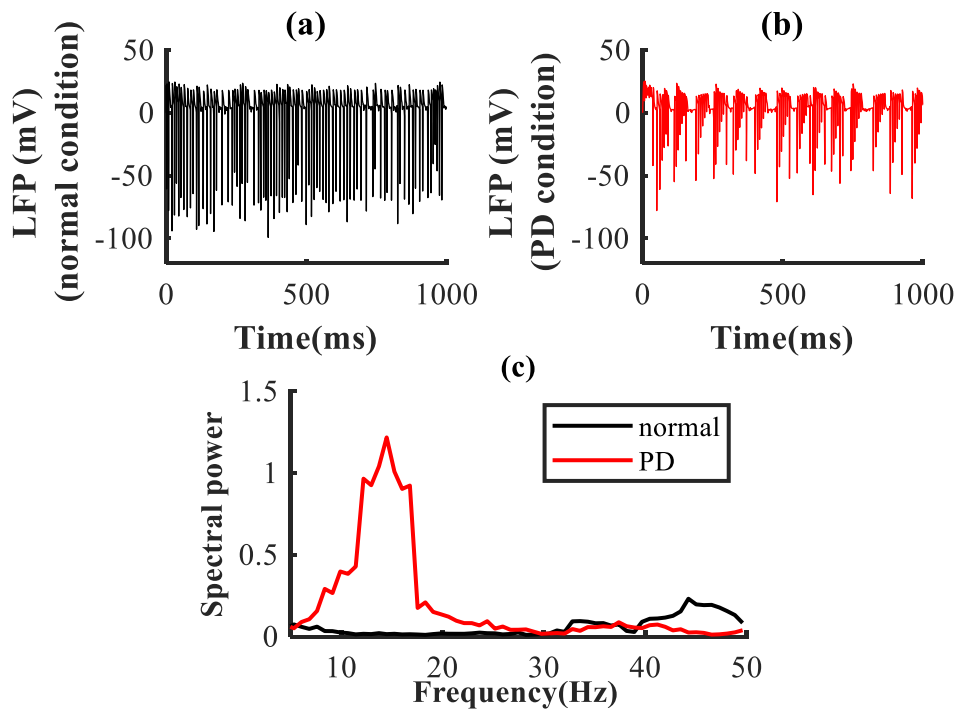

Fig. 5. The LFPs signal of GPi under (a) normal ( $p d=0$ ) and (b) PD ( $p d=1)$ conditions. (c) exihibited the power spectrum distribution within $0-50 \mathrm{~Hz}$ in normal state and PD state.
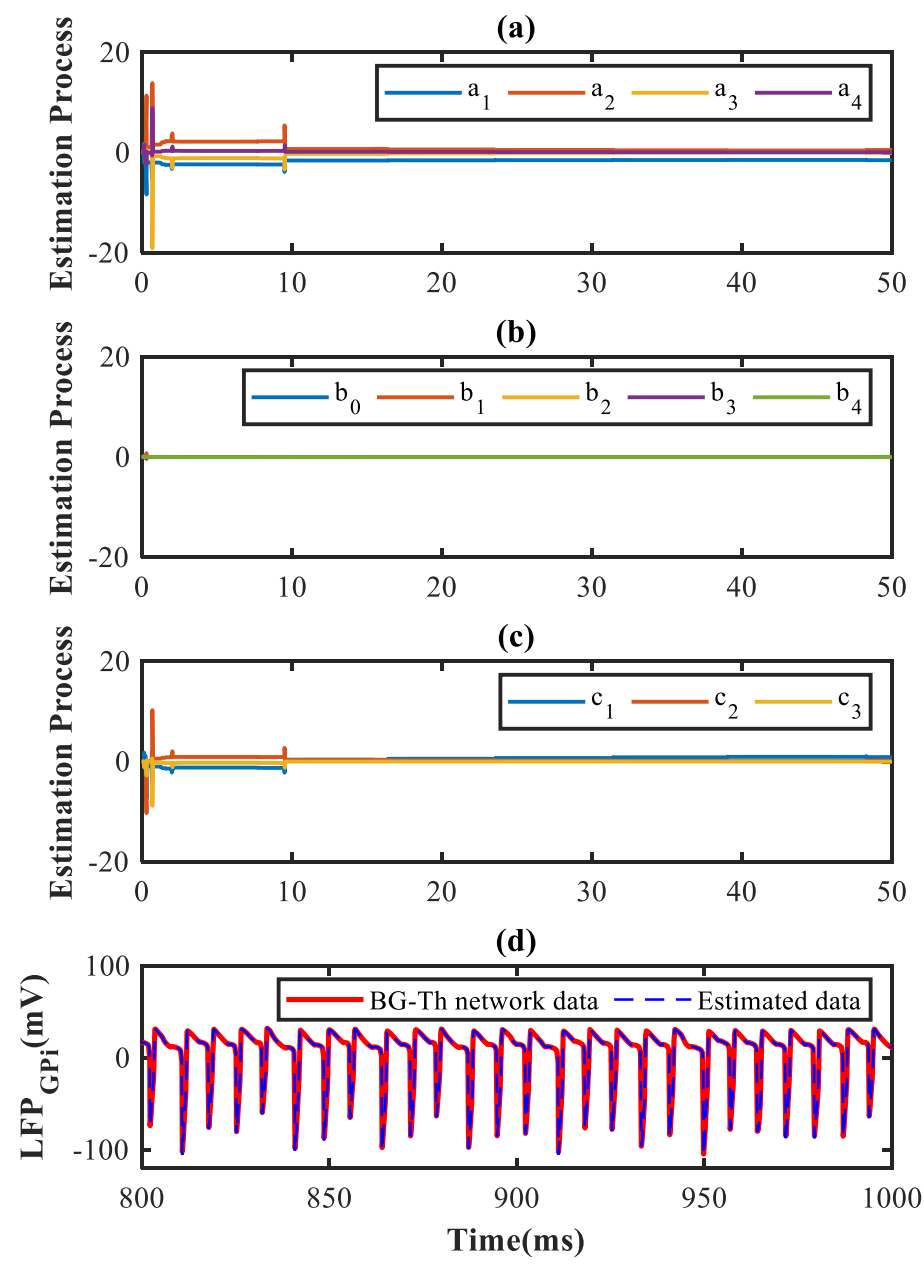

Fig. 6. (a), (b) and (c) displayed the estimation process of model parameters and (d) explicted the identification result of $\mathrm{LFP}_{G P i}$ under the input of square-wave DBS signal (with the frequency of $130 \mathrm{~Hz})$. 
(a)

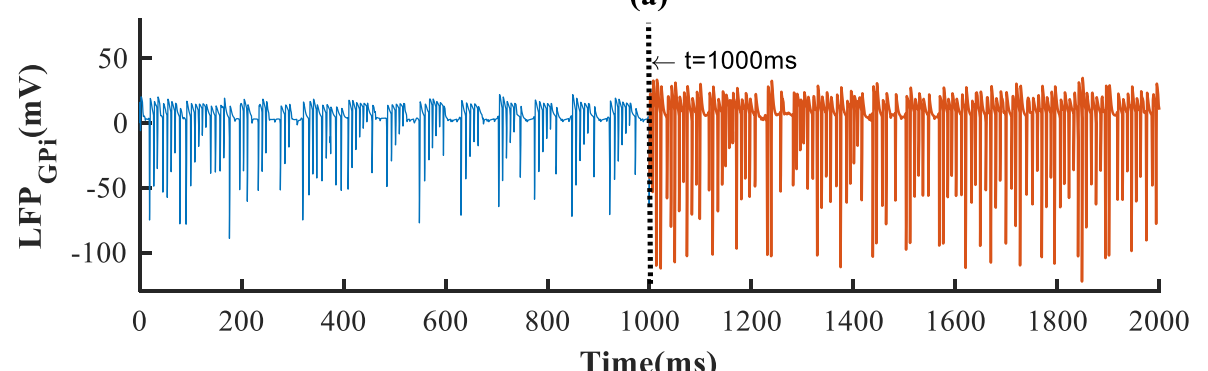

(b)
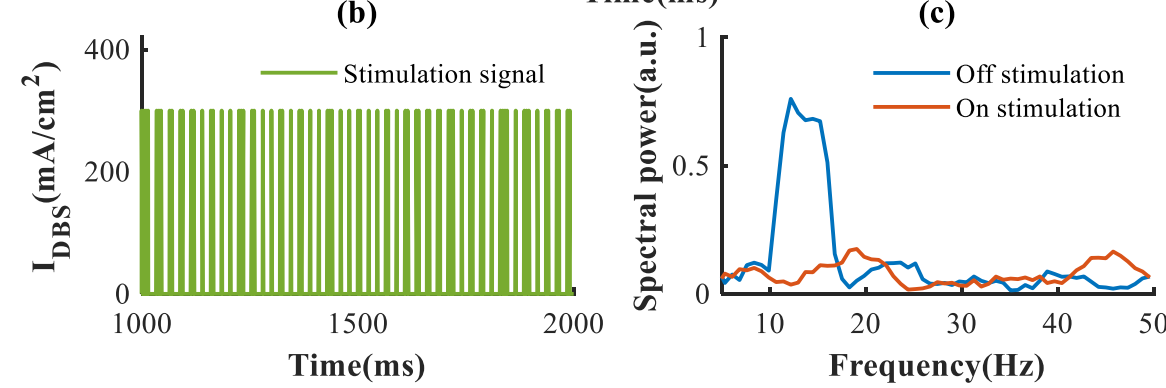

Fig. 7. Frequency modulation based on GMVC strategy. (a) was evolution of the $\mathrm{LFP}_{G P i}$ where external stimulation was exerted since $\mathrm{t}=1000 \mathrm{~ms}$. (b) Waveform of the stimulation signal $I_{\text {DBS }}$ that calculated according to the GMVC strategy. (c) Comparison of the power spectral analysis before and after the external stimulation. Weighted polynomial P, R and Q were set to 1,1 and 0.001 respectively, and $\mathrm{d}$ was set to 5 .

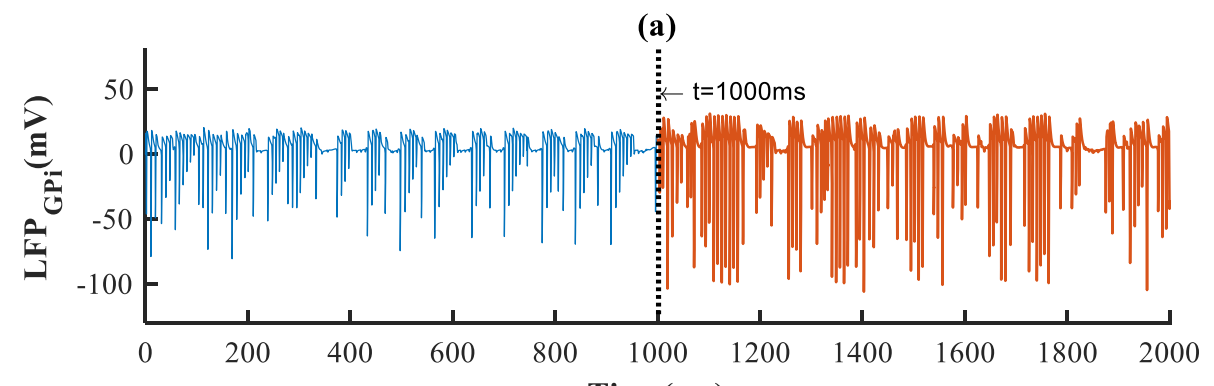

(b)
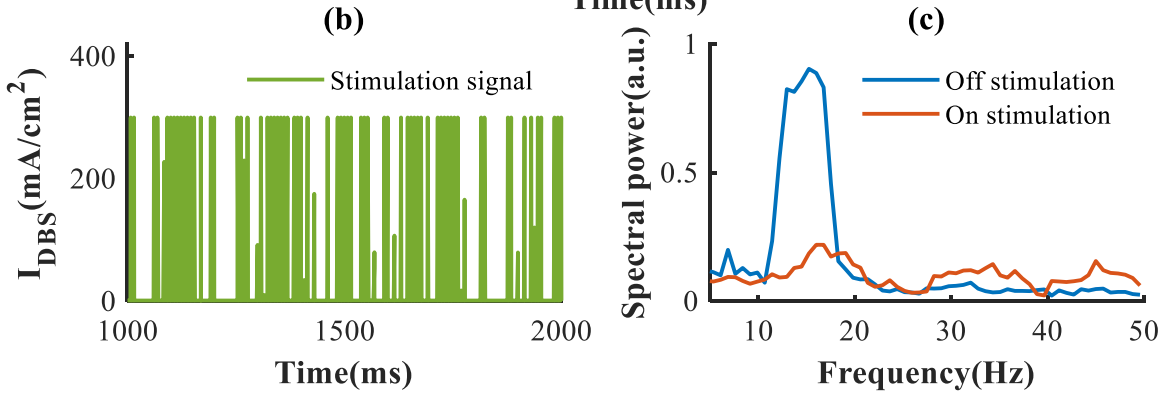

Fig. 8. Amplitude Modulation based on GMVC strategy. (a) was evolution of the $\mathrm{LFP}_{G P i}$ where external stimulation was exerted since $\mathrm{t}=1000 \mathrm{~ms}$. (b) Waveform of the stimulation signal $I_{\text {DBS }}$ that calculated according to the GMVC strategy. (c) Comparison of the power spectral analysis 
before and after the external stimulation. Controller parameters were set the same as the case of frequency modulation.

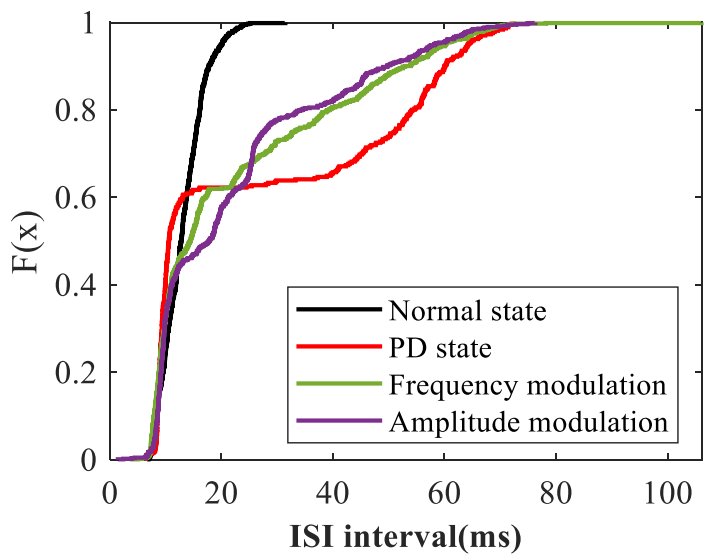

Fig. 9. Empirical cumulative distribution function of Interspike interval (ISI) distribution under normal, PD, PD condition with closed-loop frequency modulation and PD condition with closedloop amplitude modulation.
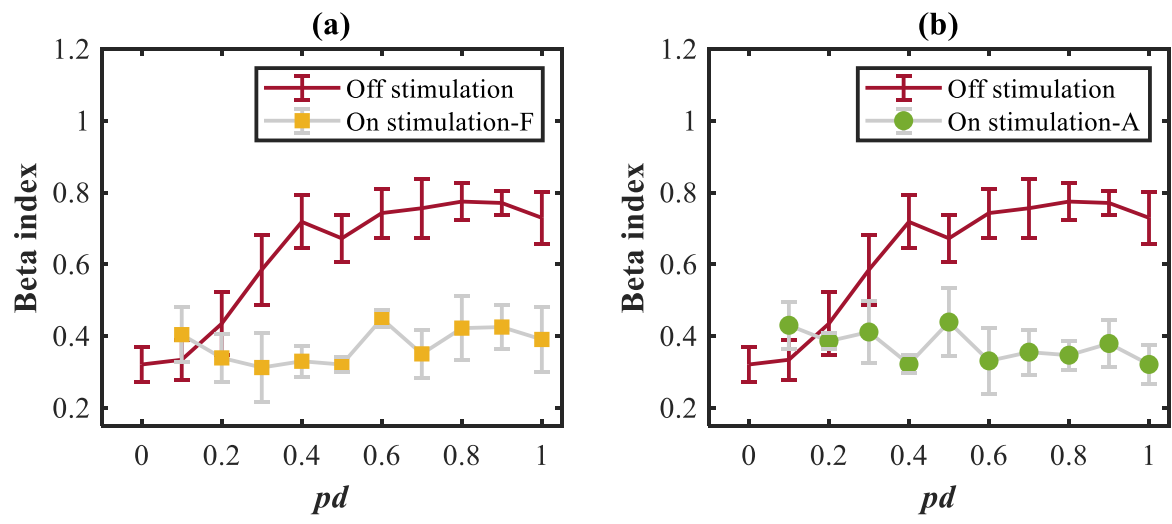

Fig. 10. Control performance of (a) frequency modulation (b) amplitude modulation under different Parkinsonian states. Standard error bars were shown for 10 simulations for each value of $p d$. 


\begin{tabular}{|c|c|c|c|}
\hline & STN & GPe(GPi) & Th \\
\hline \multicolumn{4}{|c|}{ Ionic currents } \\
\hline$I_{L}$ & $2.25(v+60)$ & $0.1(v+65)$ & $0.05(v+70)$ \\
\hline$I_{K}$ & $45 n^{4}(v+80)$ & $30 n^{4}(v+80)$ & $5(0.75(1-h))(v+75)$ \\
\hline$I_{\mathrm{Na}}$ & $37 m_{\infty}(v)^{3} h(v-55)$ & $120 m_{\infty}(v)^{3} h(v-55)$ & $3 m_{\infty}(v)^{3} h(v-50)$ \\
\hline$I_{T}$ & $0.5 a_{\infty}(v)^{3} b_{\infty}(r)^{2} v$ & $0.5 a_{\infty}(v)^{3} r v$ & $5 p_{\infty}(v)^{2} r v$ \\
\hline$I_{C a}$ & $2 c^{2}(v-140)$ & $0.15 s_{\infty}(v)^{2}(v-120)$ & -- \\
\hline$I_{A H P}$ & $20(v+80)([C a] /([C a]+15))$ & $10(v+80)([C a] /([C a]+10))$ & -- \\
\hline \multicolumn{4}{|c|}{ Gating kinetics } \\
\hline$n_{\infty}$ & $\frac{1}{1+\exp ((-v-32) / 8)}$ & $\frac{1}{1+\exp ((-v-50) / 14)}$ & -- \\
\hline$m$ & 1 & 1 & 1 \\
\hline & $\overline{1+\exp ((-v-30) / 15)}$ & $\overline{1+\exp ((-v-37) / 10)}$ & $\overline{1+\exp ((-v-37) / 7)}$ \\
\hline$h_{\infty}$ & 1 & 1 & 1 \\
\hline & $\overline{1+\exp ((v+39) / 3.1)}$ & $\overline{1+\exp ((v+58) / 12)}$ & $\overline{1+\exp ((v+41) / 4)}$ \\
\hline$a_{\infty}$ & $\frac{1}{1+\exp ((-v-63) / 7.8)}$ & $\frac{1}{1+\exp ((-v-57) / 2)}$ & -- \\
\hline$b_{\infty}$ & $\frac{1}{1+\exp ((-r+0.4) / 0.1)}-\frac{1}{1+\exp (4)}$ & -- & -- \\
\hline$r_{\infty}$ & $\frac{1}{1+\exp ((v+67) / 2)}$ & -- & $\frac{1}{1+\exp ((v+84) / 4)}$ \\
\hline$c_{\infty}$ & $1 /(1+\exp ((-v-20) / 8))$ & -- & -- \\
\hline$s_{\infty}$ & -- & $\frac{1}{1+\exp ((-v-35) / 2)}$ & -- \\
\hline$p_{\infty}$ & -- & -- & $\frac{1}{1+\exp ((-v-60) / 6.2)}$ \\
\hline$\tau_{n}$ & $1+\frac{100}{1+\exp ((v-80) / 26)}$ & $0.05+\frac{0.27}{1+\exp ((v-40) / 12)}$ & -- \\
\hline$\tau_{h}$ & $1+\frac{500}{1+\exp ((v-57) / 3)}$ & $0.05+\frac{0.27}{1+\exp ((v-40) / 12)}$ & $\frac{1}{1+\exp ((v+41) / 4)}$ \\
\hline$\tau_{r}$ & $7.1+\frac{17.5}{1+\exp ((v-68) / 2.2)}$ & -- & $\frac{0.15}{28+\exp ((-v-25) / 10.5)}$ \\
\hline$\tau_{c}$ & $1+\frac{10}{1+\exp ((v+80) / 26)}$ & -- & -- \\
\hline
\end{tabular}


Figures

(a)

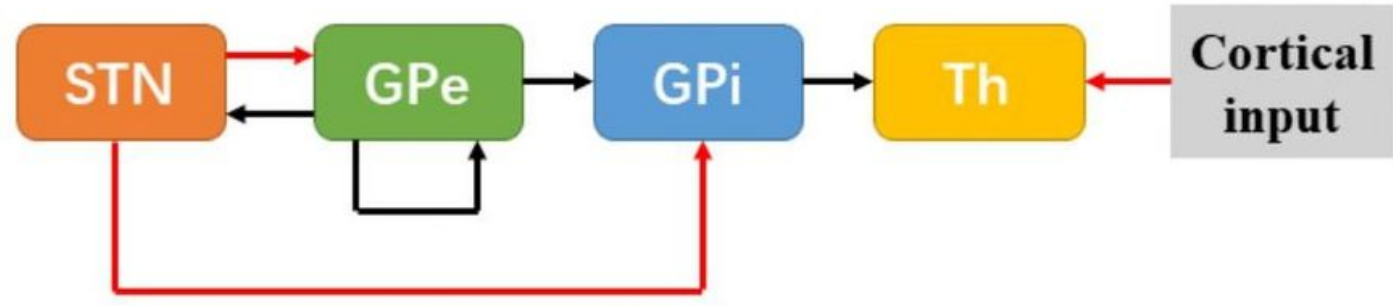

(b)

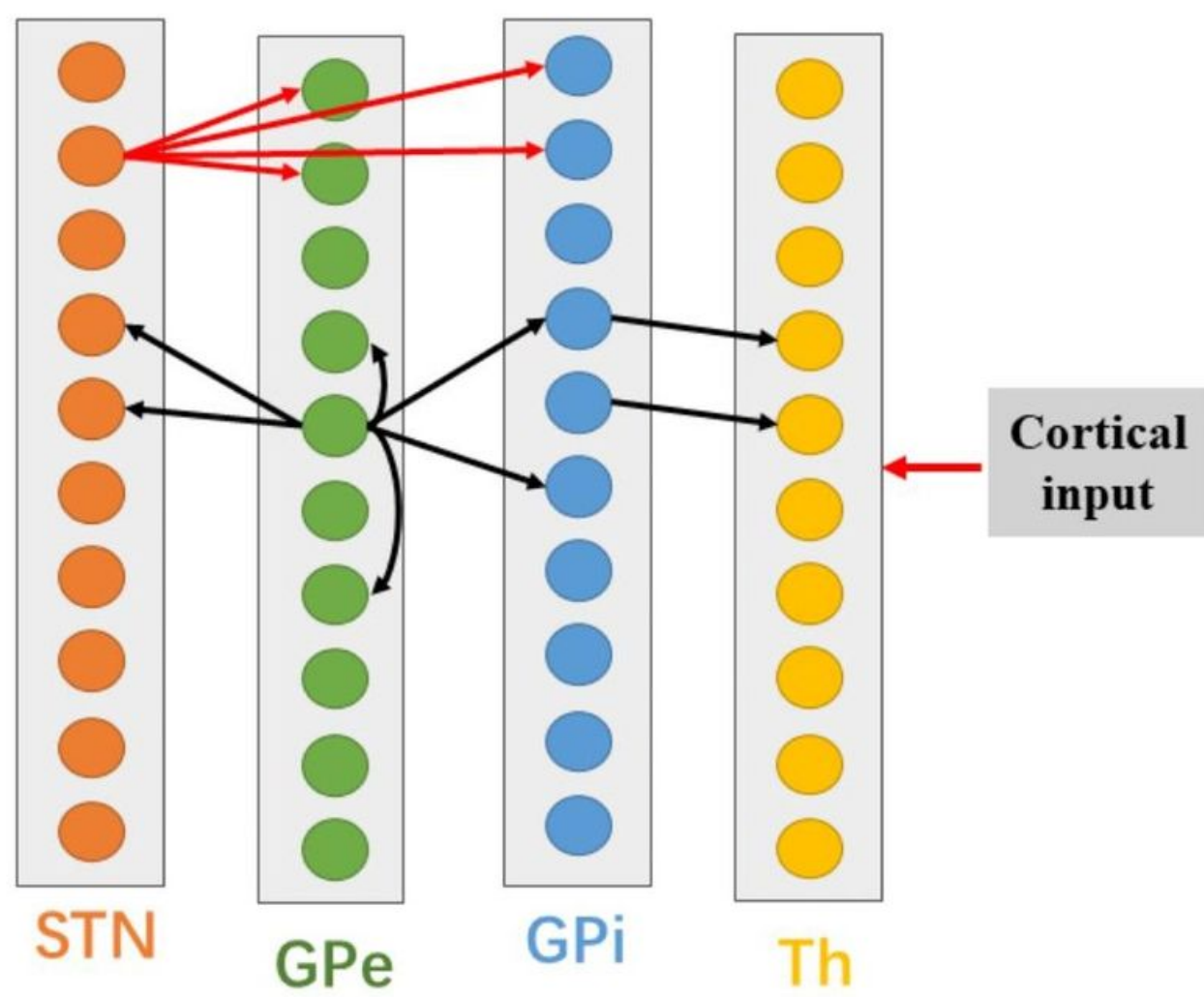

Figure 1

BG-Th network model. (a) indicated the network layout and (b) depicted details of synaptic connections among STN, GPe, GPi and Th nucleus within the network, where red arrows denote excitatory connections andblack arrows denote inhibitory connections. 


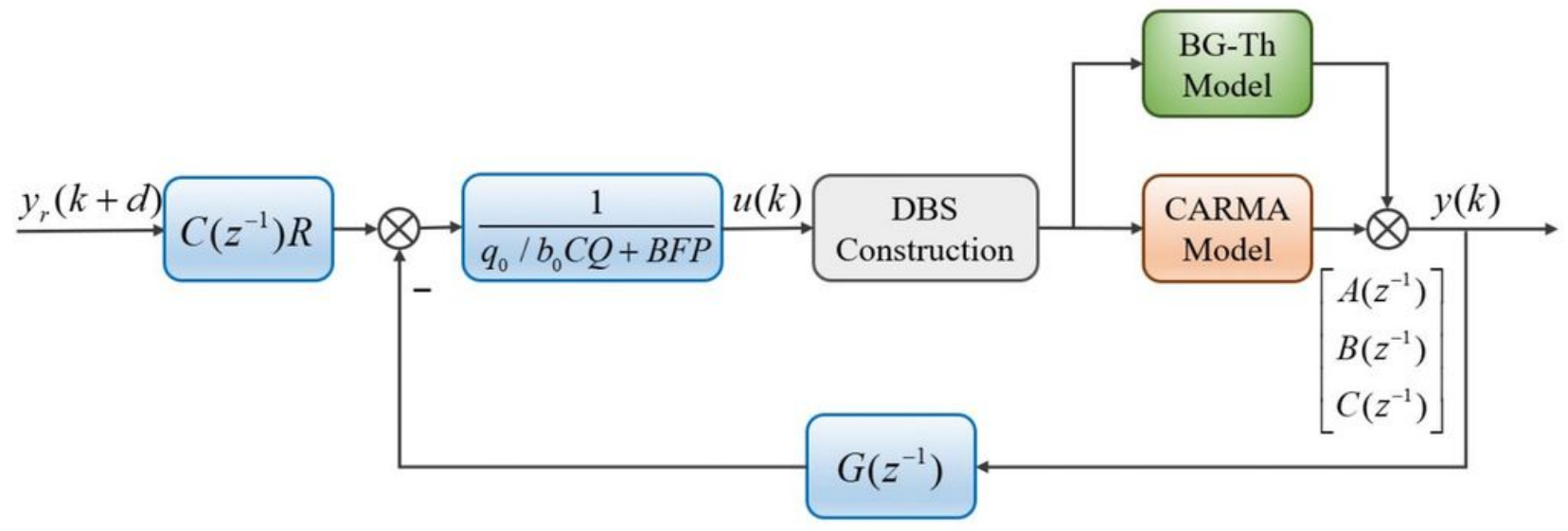

Figure 2

The diagram of CARMA model-based generalized minimum variance control system
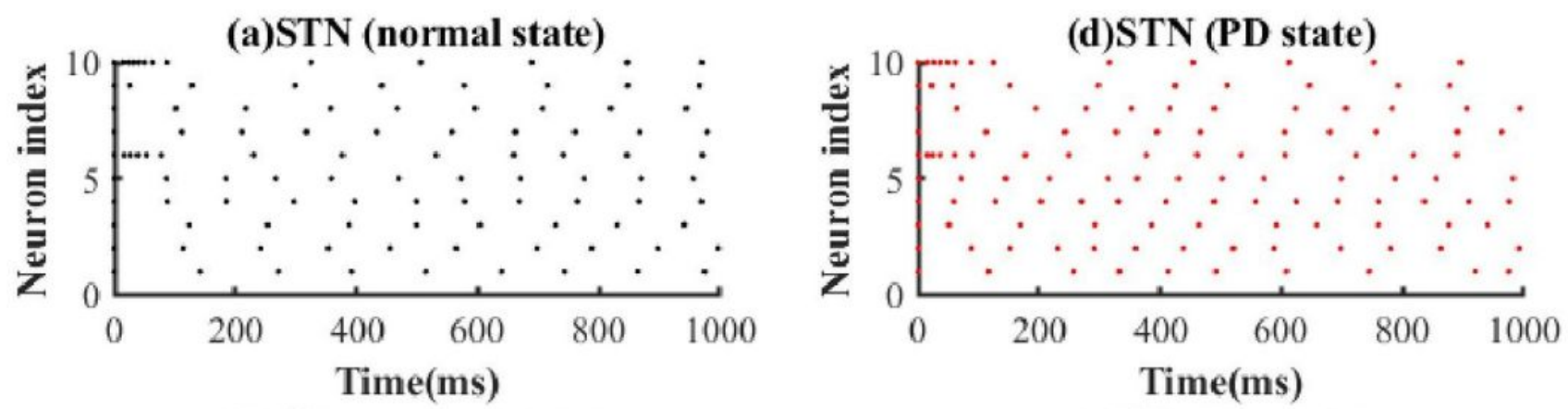

(b)GPe (normal state)

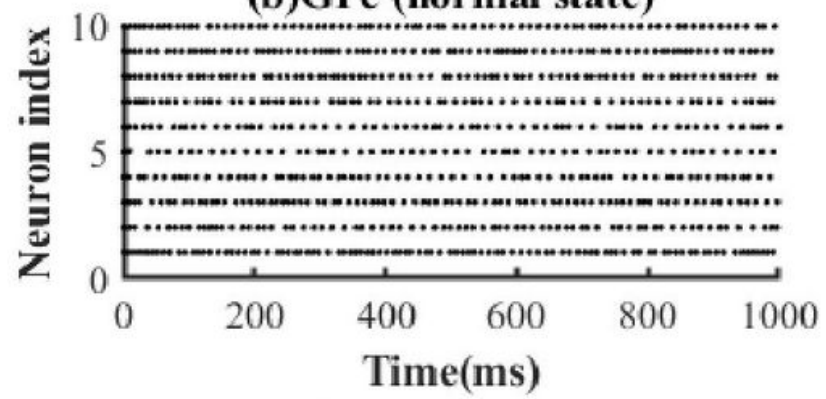

(e)GPe (PD state)

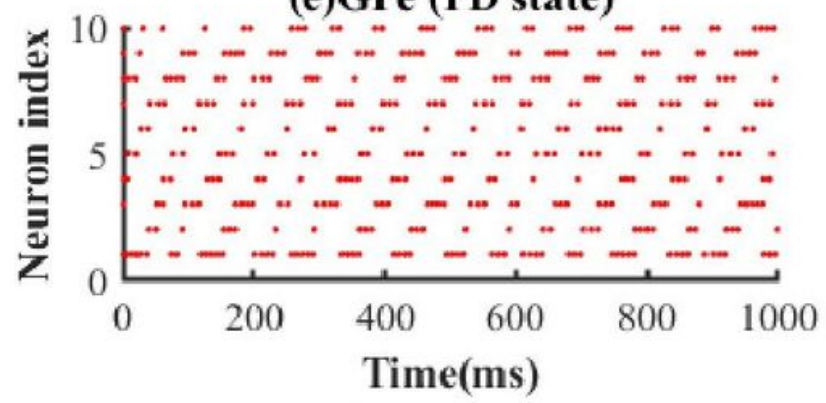

(c)GPi (normal state)
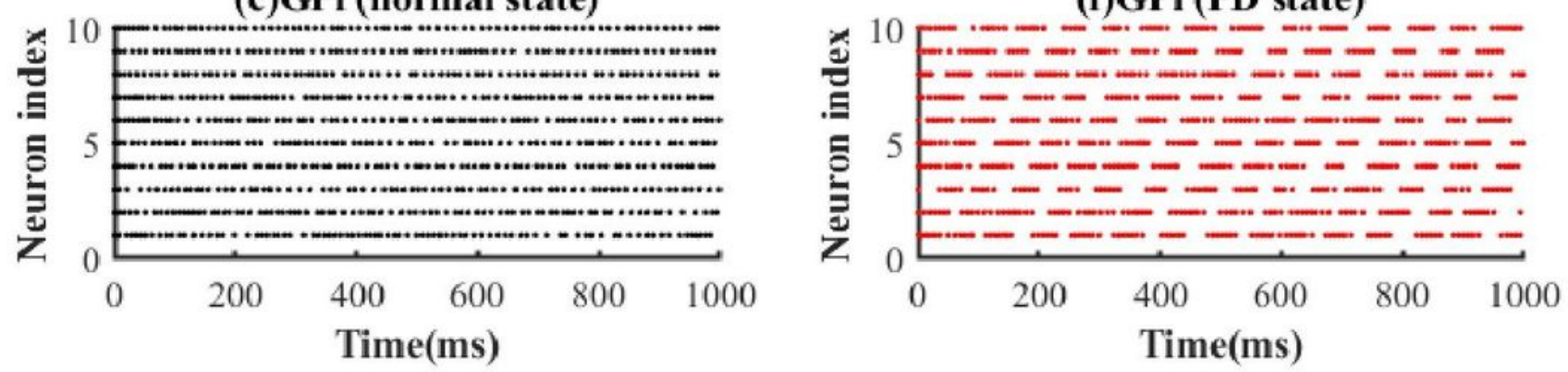

Figure 3 
Firing rastergrams of STN, GPe and GPi neurons under normal ((a), (b) and (c)) condition and PD ((d), (e) and (f)) condition.

(a)

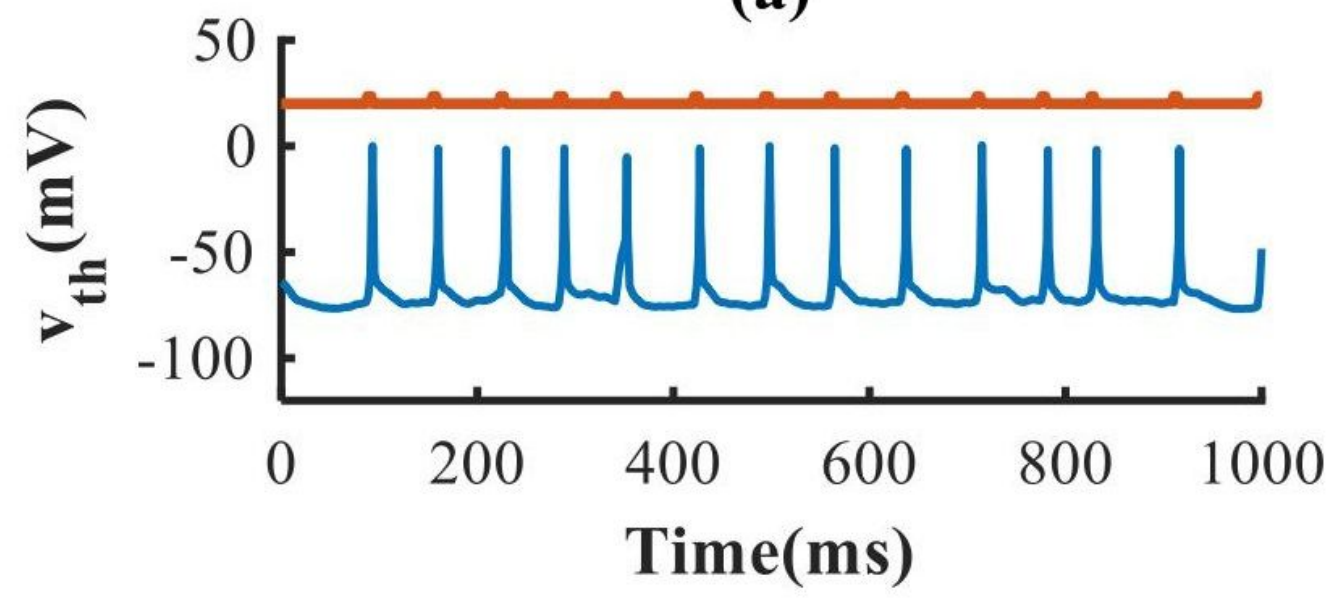

(b)

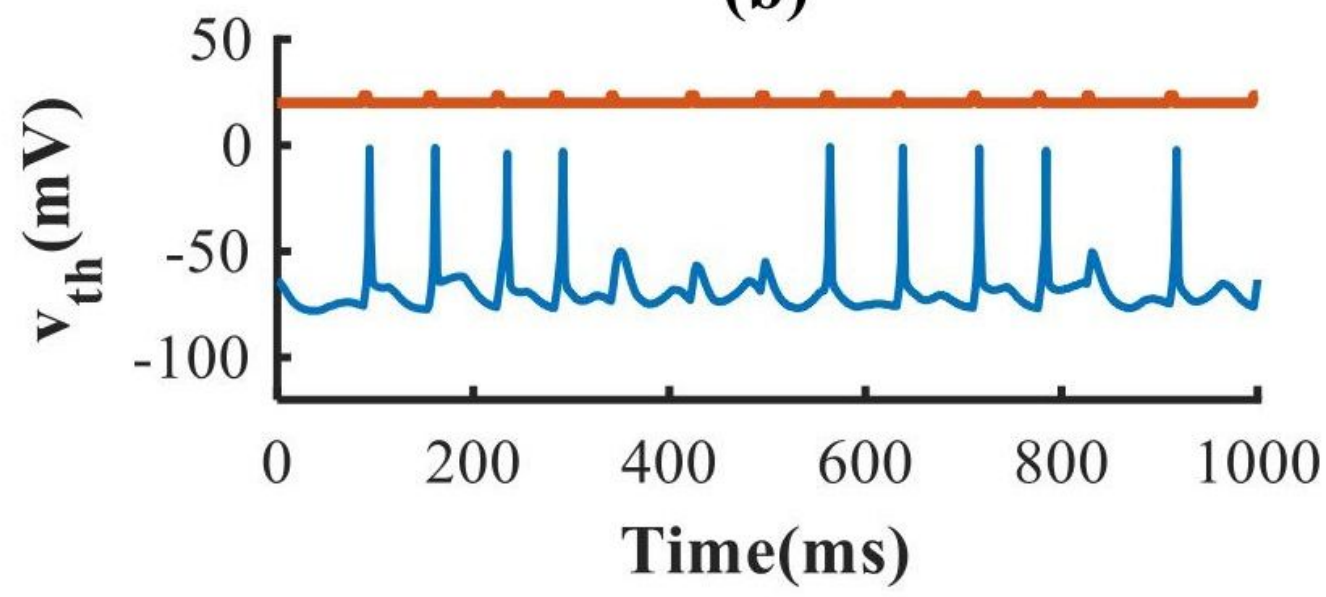

Figure 4

Membrane potential traces of the Th neurons. Orange pulse trains denoted excitatory cortex input signals, blue traces in upper and bottom pannels represented the normal condition (a) and the PD condition (b), respectively. 

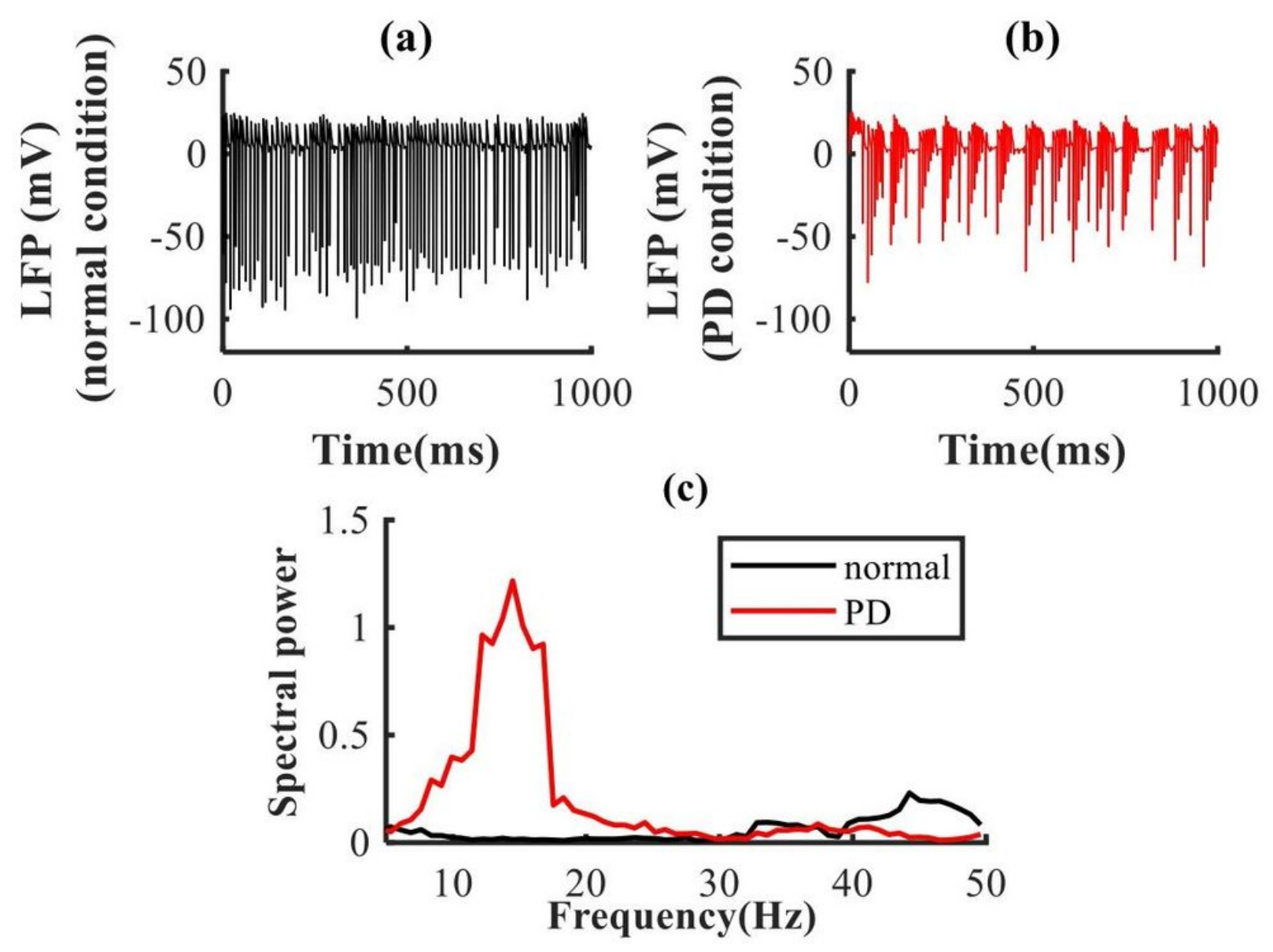

Figure 5

The LFPs signal of GPi under (a) normal ( $p d=0$ ) and (b) PD ( $p d=1)$ conditions. (c) exihibited the power spectrum distribution within $0-50 \mathrm{~Hz}$ in normal state and PD state 

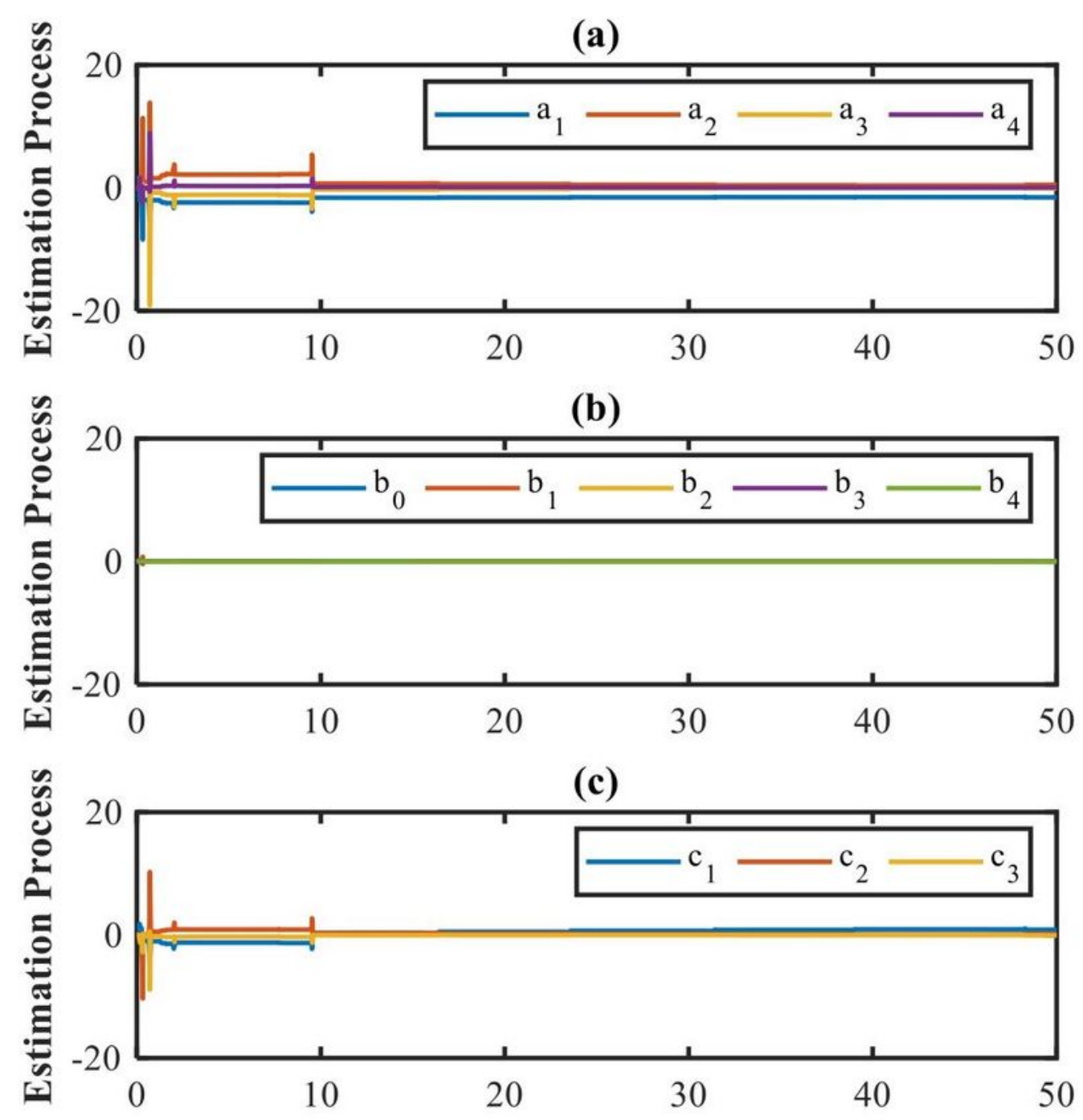

(d)

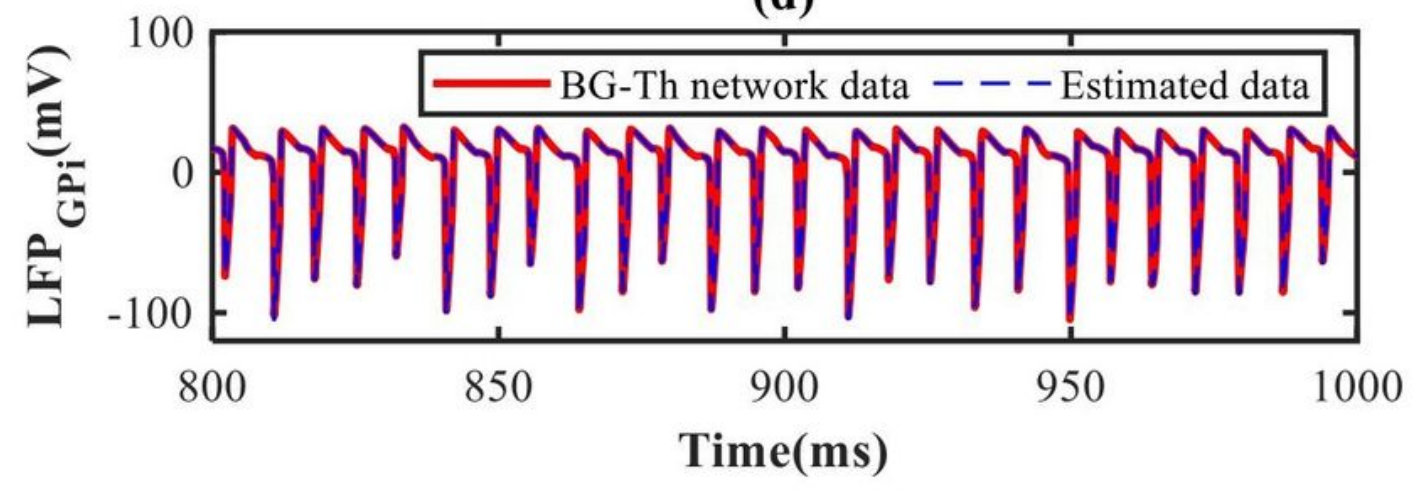

Figure 6

(a), (b) and (c) displayed the estimation process of model parameters and (d) explicted the identification result of LFPGPi under the input of square-wave DBS signal (with the frequency of $130 \mathrm{~Hz}$ ). 
(a)
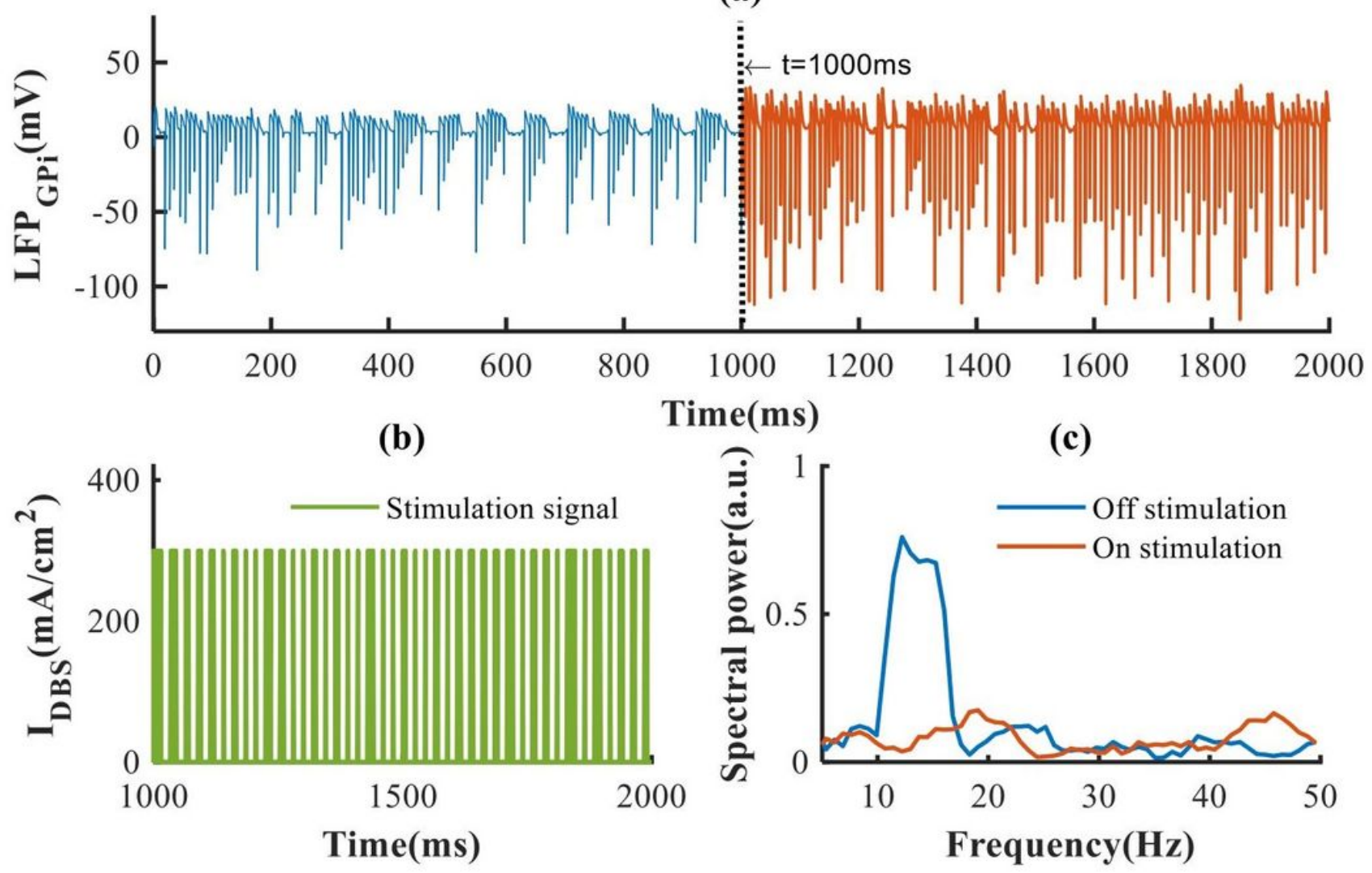

Figure 7

Frequency modulation based on GMVC strategy. (a) was evolution of the LFPGPi where external stimulation was exerted since $t=1000 \mathrm{~ms}$. (b) Waveform of the stimulation signal DBS I that calculated according to the GMVC strategy. (c) Comparison of the power spectral analysis before and after the external stimulation. Weighted polynomial $\mathrm{P}, \mathrm{R}$ and $\mathrm{Q}$ were set to 1,1 and 0.001 respectively, and $\mathrm{d}$ was set to 5 . 
(a)
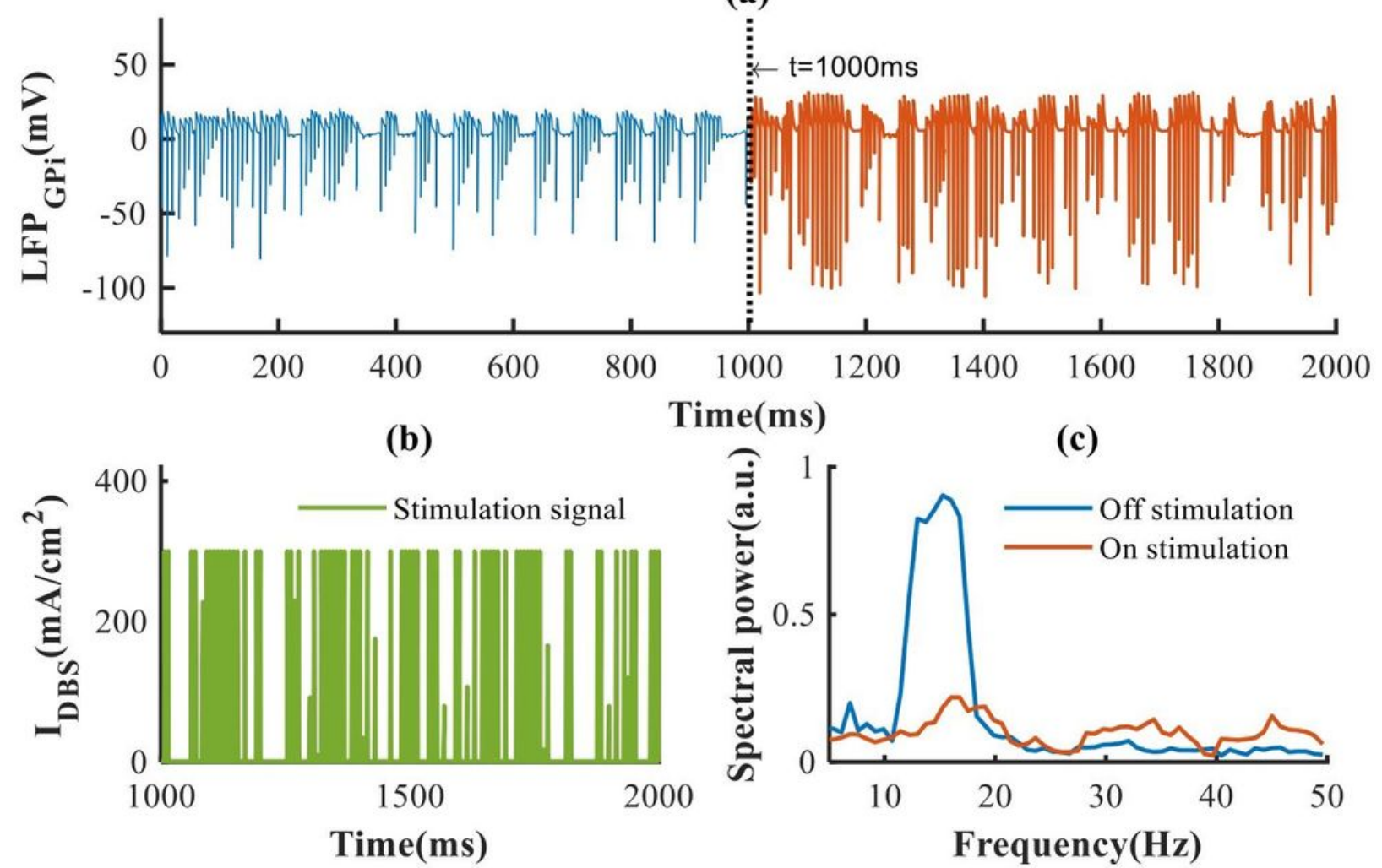

Figure 8

Amplitude Modulation based on GMVC strategy. (a) was evolution of the LFPGPi where external stimulation was exerted since $t=1000 \mathrm{~ms}$. (b) Waveform of the stimulation signal DBS I that calculated according to the GMVC strategy. (c) Comparison of the power spectral analysis 22 before and after the external stimulation. Controller parameters were set the same as the case of frequency modulation. 


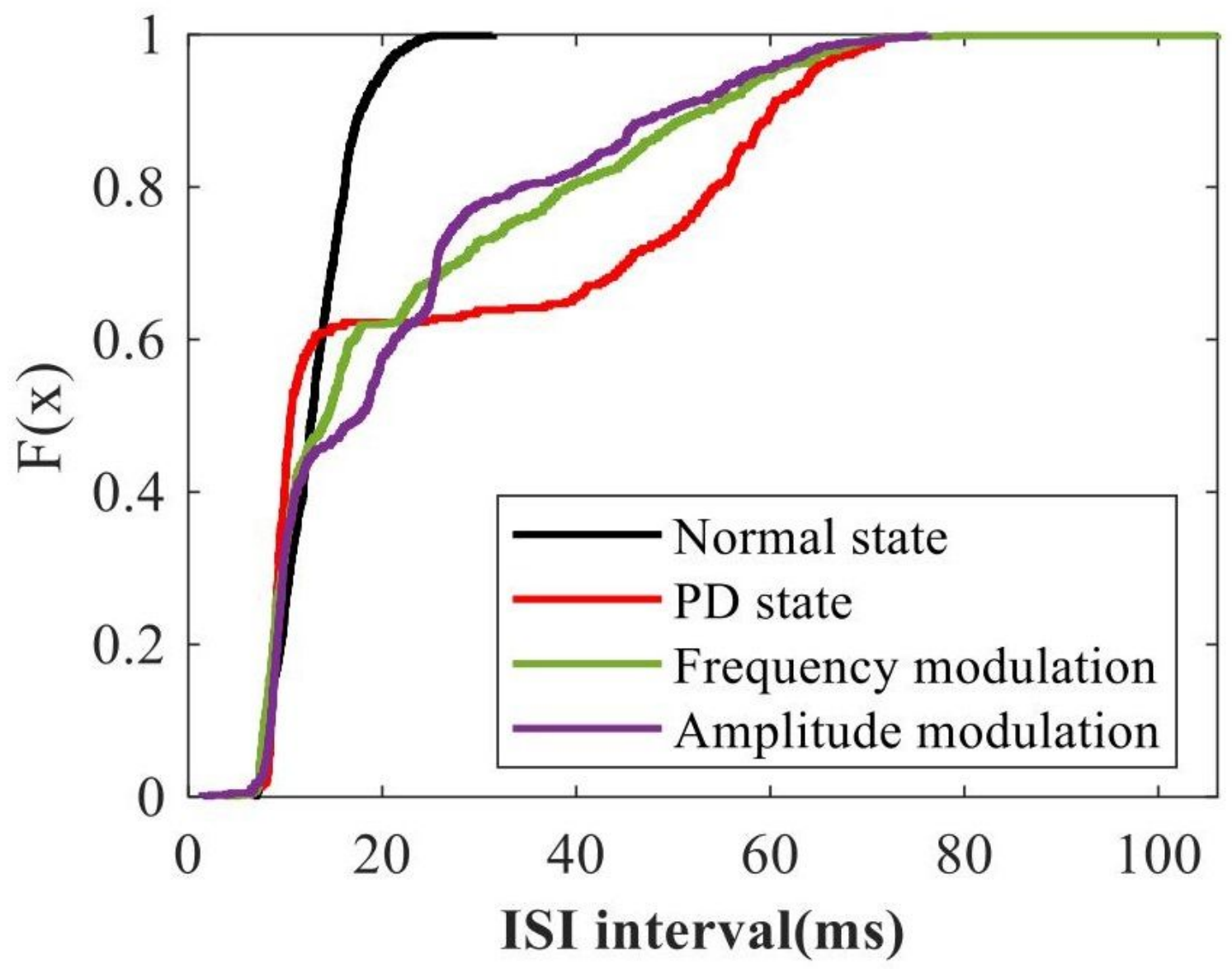

Figure 9

Empirical cumulative distribution function of Interspike interval (ISI) distribution under normal, PD, PD condition with closed-loop frequency modulation and PD condition with closed冈loop amplitude modulation.

(a)

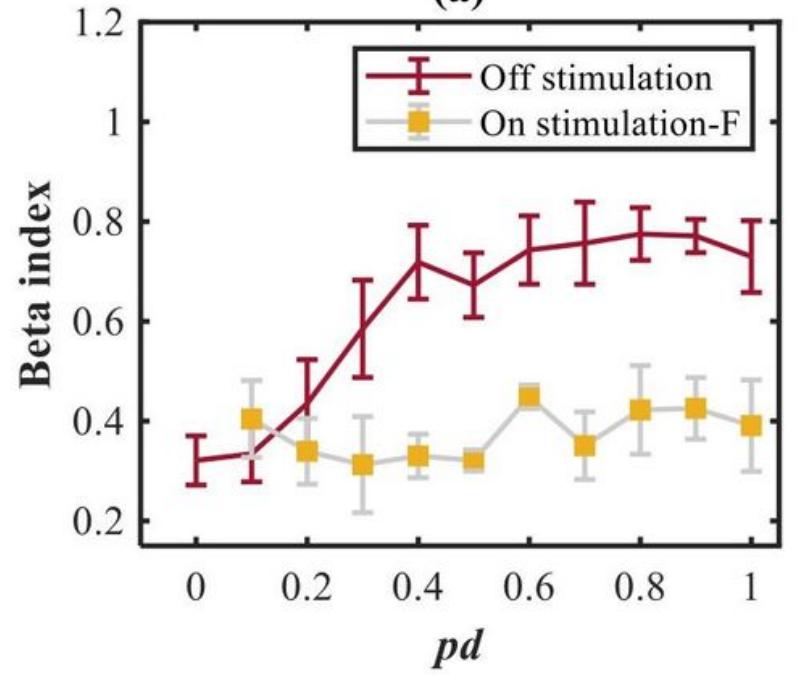

(b)

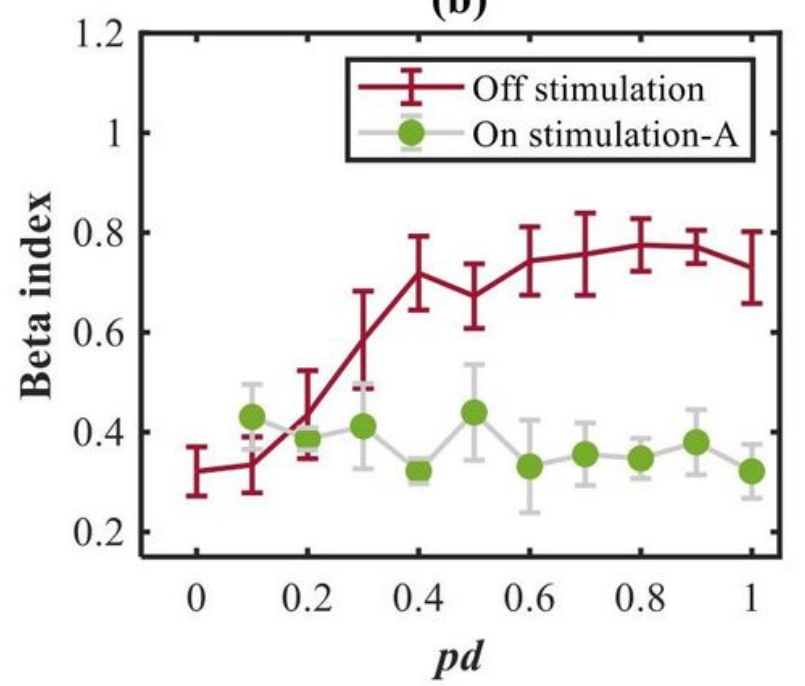


Figure 10

Control performance of (a) frequency modulation (b) amplitude modulation under different Parkinsonian states. Standard error bars were shown for 10 simulations for each value of $\mathrm{pd}$ 